\title{
Asymptotic Theory of Quantum Channel Estimation
}

\author{
Sisi Zhou $\oplus^{1,2,{ }^{*}}$ and Liang Jiang $\oplus^{2}$ \\ ${ }^{1}$ Department of Physics, Yale University, New Haven, Connecticut 06511, USA \\ ${ }^{2}$ Pritzker School of Molecular Engineering, The University of Chicago, Illinois 60637, USA
}

(Received 23 March 2020; revised 20 December 2020; accepted 26 February 2021; published 16 March 2021)

\begin{abstract}
The quantum Fisher information (QFI), as a function of quantum states, measures the amount of information that a quantum state carries about an unknown parameter. The (entanglement-assisted) QFI of a quantum channel is defined to be the maximum QFI of the output state assuming an entangled input state over a single probe and an ancilla. In quantum metrology, people are interested in calculating the QFI of $N$ identical copies of a quantum channel when $N \rightarrow \infty$, which is called the asymptotic QFI. Over the years, researchers found various types of upper bounds of the asymptotic QFI, but they were proven achievable only in several specific situations. It was known that the asymptotic QFI of an arbitrary quantum channel grows either linearly or quadratically with $N$. Here we show that a simple criterion can determine whether the scaling is linear or quadratic. In both cases, the asymptotic QFI and a quantum error correction protocol to achieve it are computable via a semidefinite program. When the scaling is quadratic, the Heisenberg limit, a feature of noiseless quantum channels, is recovered. When the scaling is linear, we show that the asymptotic QFI is still in general larger than $N$ times the single-channel QFI and, furthermore, that sequential estimation strategies provide no advantage over parallel ones.
\end{abstract}

DOI: 10.1103/PRXQuantum.2.010343

\section{INTRODUCTION}

Quantum metrology studies parameter estimation in a quantum system [1-5]. Usually, a quantum probe interacts with a physical system and the experimentalist performs measurements on the final probe state and infers the value of the unknown parameter(s) in the system from the measurement outcomes. It has wide applications in frequency spectroscopy [6-9], gravitational-wave detectors [10-13], and other high-precision measurements [14-18].

The quantum Fisher information (QFI), which is inversely proportional to the minimum estimation variance, characterizes the amount of information a quantum state carries about an unknown parameter [19-22]. To explore the fundamental limit on parameter estimation, we usually consider the situation where the number of quantum channels $N$ (or the probing time $t$ ) is large. The Heisenberg limit (HL), an $O\left(N^{2}\right)$ [or $O\left(t^{2}\right)$ ] scaling of the QFI, is the ultimate estimation limit allowed by quantum mechanics. It could be obtained, for example, using Greenberger-Horne-Zeilinger (GHZ) states in noiseless systems $[9,23]$. On the other hand, the standard

\footnotetext{
*sisi.zhou26@gmail.com
}

Published by the American Physical Society under the terms of the Creative Commons Attribution 4.0 International license. Further distribution of this work must maintain attribution to the author(s) and the published article's title, journal citation, and DOI. quantum limit (SQL), an $O(N)$ [or $O(t)$ ] scaling of the QFI, usually appears in noisy systems and could be achieved using product states. Much work has been done towards determining whether or not the HL is achievable for a given quantum channel and some necessary conditions were derived [24-37].

In general, the asymptotic QFI of a quantum system, i.e., the QFI in the $N \rightarrow \infty$ limit [29], follows either the HL or the SQL and there was not a unified approach to determine the scaling. For quantum channels where the scalings are known, it is also crucial to understand how to achieve the asymptotic QFI. For example, for unitary channels, the HL is achievable and a GHZ state in the multipartite two-level systems consisting of the lowest and highest energy states is optimal [23]. Under the effect of noise, a variety of quantum strategies were also proposed to enhance the QFI $[8,10,38-52]$, but no conclusions for general quantum channels were drawn. One natural question to ask is whether entanglement between probes can improve the QFI. For example, when estimating the noise parameter in the dissipative low-noise channels $[53,54]$ or teleportation-covariant channels [5558] (e.g., Pauli or erasure channels), the asymptotic QFI follows the SQL and is achievable using only product states. However, when estimating the phase parameter in dephasing channels, although the HL is still not achievable, product states are no longer optimal and the asymptotic QFI is then achievable using spin-squeezed states $[8,31,39]$. 
Given a quantum channel, we aim to answer the following two important questions: how to determine whether the HL is achievable, and in both cases, how to find a metrological protocol achieving the asymptotic QFI? In this paper, we answer these two open problems in the setting of entanglement-assisted channel estimation by providing an optimal quantum error correction (QEC) metrological protocol that entangles both the probe and a clean ancillary system. QEC has been a powerful tool widely used in quantum computing and quantum communication to protect quantum information from noise [59-62]. In quantum metrology, QEC is also useful in protecting a quantum signal from quantum noise [32-34,63-76]. Here is a typical example: when a qubit is subject to a Pauli- $Z$ signal and a Pauli- $X$ noise, the QFI follows the SQL if no quantum control is added, but the HL is recoverable using fast and frequent QEC [63-68]. The result could be generalized to any system with a signal Hamiltonian and Markovian noise [33,34]. These QEC protocols, however, can only estimate Hamiltonian parameters and all rely on fast and frequent quantum operations that have limited practical applications.

In this paper, we construct a two-dimensional QEC protocol that reduces every quantum channel to a single-qubit dephasing channel where both the phase and the noise parameter could vary with respect to the unknown parameter. We first identify the asymptotic QFI for all singlequbit dephasing channels (where the unknown parameter is encoded in both the noise and phase parameters) and then show that the asymptotic QFI of the logical dephasing channel is no smaller than that of the original quantum channel after optimizing over the encoding and the recovery channel, proving the sufficiency of our QEC protocol. Using the above proof strategy, we obtain the asymptotic theory of quantum channel estimation, closing a longstanding open question in theoretical quantum metrology. We also push one step further towards achieving the ultimate estimation limit in practical quantum sensing experiments by providing efficiently computable asymptotic QFI and corresponding optimal estimation protocols.

\section{OVERVIEW}

\section{A. Preliminaries}

The quantum Cramér-Rao bound is a lower bound of the estimation precision [19-22],

$$
\delta \omega \geq \frac{1}{\sqrt{N_{\operatorname{expr}} F\left(\rho_{\omega}\right)}},
$$

where $\omega$ is an unknown real parameter to be estimated, $\delta \omega$ is the standard deviation of any unbiased estimator of $\omega$, $N_{\text {expr }}$ is the number of repeated experiments, and $F\left(\rho_{\omega}\right)$ is the QFI of the state $\rho_{\omega}$. The quantum Cramér-Rao bound is saturable asymptotically $\left(N_{\text {expr }} \gg 1\right)$ using maximum likelihood estimators [77,78]. Therefore, the QFI is a good measure of the amount of information a quantum state $\rho_{\omega}$ carries about an unknown parameter. It is defined by $F\left(\rho_{\omega}\right)=\operatorname{Tr}\left(L^{2} \rho_{\omega}\right)$, where $L$ is a Hermitian operator called the symmetric logarithmic derivative (SLD) satisfying

$$
\dot{\rho}_{\omega}=\frac{1}{2}\left(\rho_{\omega} L+L \rho_{\omega}\right),
$$

where $\star$ denotes $\partial \star / \partial \omega$. We use $L_{A}[B]$ to represent Hermitian operators satisfying $B=\frac{1}{2}(L A+A L)$. Here $L=$ $L_{\rho_{\omega}}\left[\dot{\rho}_{\omega}\right]$. The QFI could also be equivalently defined through purification $[24,29]$ :

$$
F\left(\rho_{\omega}\right)=4 \min _{\left|\psi_{\omega}\right\rangle: \operatorname{Tr}_{E}\left(\left|\psi_{\omega}\right\rangle\left\langle\psi_{\omega}\right|\right)=\rho_{\omega}}\left\langle\dot{\psi}_{\omega} \mid \dot{\psi}_{\omega}\right\rangle .
$$

Here $\rho_{\omega} \in \mathfrak{S}\left(\mathcal{H}_{\mathcal{P}}\right), \quad\left|\psi_{\omega}\right\rangle \in \mathfrak{S}\left(\mathcal{H}_{\mathcal{P}} \otimes \mathcal{H}_{E}\right), \mathcal{H}_{\mathcal{P}}$ is the probe system that we assume to be finite dimensional, $\mathcal{H}_{E}$ is an arbitrarily large environment, and $\mathfrak{S}(\star)$ denotes the set of density operators in $\star$.

We consider a quantum channel $\mathcal{E}_{\omega}(\rho)=\sum_{i=1}^{r} K_{i} \rho K_{i}^{\dagger}$, where $r$ is the rank of the channel. The entanglementassisted QFI of $\mathcal{E}_{\omega}$ [see Fig. 1(a)] is defined by [24,29]

$$
\mathfrak{F}_{1}\left(\mathcal{E}_{\omega}\right):=\max _{\rho \in \mathfrak{S}\left(\mathcal{H}_{\mathcal{P}} \otimes \mathcal{H}_{\mathcal{A}}\right)} F\left[\left(\mathcal{E}_{\omega} \otimes \mathbb{1}\right)(\rho)\right] .
$$

Here we utilize the entanglement between the probe and an arbitrarily large ancillary system $\mathcal{H}_{\mathcal{A}}$. We omit the word "entanglement assisted" or "ancilla assisted" in the definitions below for simplicity. Practically, the ancilla should be a quantum system with a long coherence time, e.g., nuclear spins [67] or any QEC-protected system [34]. The ancilla also helps simplify the complicated calculation of the QFI. The convexity of QFI implies that the optimal input state is always pure. Using the purification-based definition of the QFI [Eq. (3)], we have [24,28,29]

$$
\begin{gathered}
\mathfrak{F}_{1}\left(\mathcal{E}_{\omega}\right)=4 \max _{\rho \in \mathfrak{S}(\mathcal{H} \mathcal{P})} \min _{\text {for all } u \text { such that } u^{\dagger} u=I} \operatorname{Tr}\left(\rho \dot{\mathbf{K}}^{\prime \dagger} \dot{\mathbf{K}}^{\prime}\right) \\
=4 \min _{\substack{\mathbf{K}^{\prime}=u \mathbf{K} \\
\text { for all } u \text { such that } u^{\dagger} u=I}}\left\|\dot{\mathbf{K}}^{\prime \dagger} \dot{\mathbf{K}}^{\prime}\right\|=4 \min _{h \in \mathbb{H}_{r}}\|\alpha\|,
\end{gathered}
$$

where $\|\cdot\|$ is the operator norm, $\mathbb{H}_{r}$ is the space of $r \times r$ Hermitian matrices, and $\mathbf{K}=\left(K_{1}, \ldots, K_{r}\right)^{T} . \mathbf{K}^{\prime}=$ $\left(K_{1}^{\prime}, \ldots, K_{r}^{\prime}\right)^{T}=u \mathbf{K}$ represents all possible Kraus representations of $\mathcal{E}_{\omega}$ via isometric transformations $u$ [24]. Let $h=i u^{\dagger} \dot{u}$ and $\alpha=\dot{\mathbf{K}}^{\prime \dagger} \dot{\mathbf{K}}^{\prime}=(\dot{\mathbf{K}}-i h \mathbf{K})^{\dagger}(\dot{\mathbf{K}}-i h \mathbf{K})$. The minimization is performed over arbitrary Hermitian operator $h$ in $\mathbb{C}^{r \times r}$ [28]. Any purification of the optimal $\rho$ in Eq. (5) is an optimal input state in $\mathcal{H}_{\mathcal{P}} \otimes \mathcal{H}_{\mathcal{A}}$ and it in general depends on the true value of $\omega$ and should be chosen adaptively throughout the experiment $[79,80]$. The QFI $\mathfrak{F}_{1}\left(\mathcal{E}_{\omega}\right)$ 
(a)

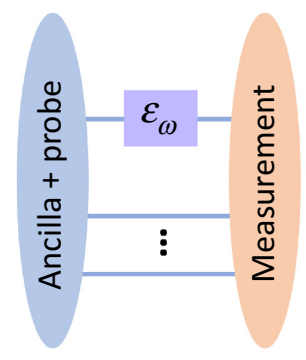

(b)

(c)

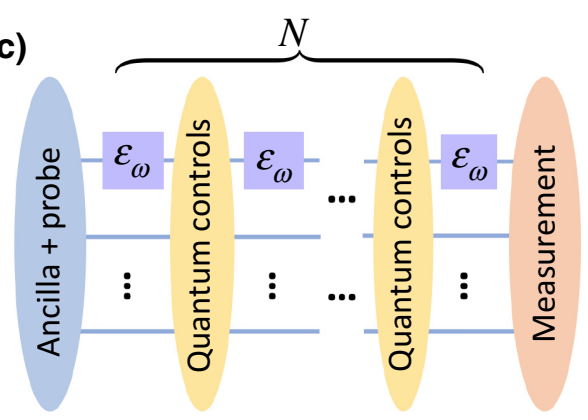

FIG. 1. (a) The single-channel QFI $\mathfrak{F}_{1}\left(\mathcal{E}_{\omega}\right)=\max _{\rho} F\left[\left(\mathcal{E}_{\omega} \otimes\right.\right.$ $\mathbb{1})(\rho)$ ]. The ancillary system is assumed to be arbitrarily large. (b) Parallel strategies. We have $\mathfrak{F}_{N}\left(\mathcal{E}_{\omega}\right)=\mathfrak{F}_{1}\left(\mathcal{E}_{\omega}^{\otimes N}\right)=$ $\max _{\rho} F\left[\left(\mathcal{E}_{\omega}^{\otimes N} \otimes \mathbb{1}\right)(\rho)\right]$ for $N$ identical copies of $\mathcal{E}_{\omega}$. (c) Sequential strategies. Let $F_{N}\left(\mathcal{E}_{\omega}, \mathscr{S}\right)$ be the QFI of the output state, given a sequential strategy $\mathscr{S}$ that contains both an input state and quantum controls acting between $\mathcal{E}_{\omega}$. Here $\mathfrak{F}_{N}^{(\text {seq) }}\left(\mathcal{E}_{\omega}\right)=$ $\max _{\mathscr{S}} F_{N}\left(\mathcal{E}_{\omega}, \mathscr{S}\right)$ is the optimal QFI maximized over all sequential strategies; $\mathfrak{F}_{N}^{\text {(seq) }}\left(\mathcal{E}_{\omega}\right) \geq \mathfrak{F}_{N}\left(\mathcal{E}_{\omega}\right)$.

can be found via a semidefinite program (SDP) [28,29], as well as the optimal input state (see Appendix F). As an example, we show in Appendix $G$ that, when viewing the ancilla as a lossless arm in the Mach-Zehnder interferometer $[81,82]$, the SDP in Appendix F leads to a SDP solving for the optimal input state with a definite photon number.

Consider $N$ identical copies of the quantum channel $\mathcal{E}_{\omega}$ [24,28] [see Fig. 1(b)]. Let

$$
\mathfrak{F}_{N}\left(\mathcal{E}_{\omega}\right):=\mathfrak{F}_{1}\left(\mathcal{E}_{\omega}^{\otimes N}\right)=\max _{\rho} F\left[\left(\mathcal{E}_{\omega}^{\otimes N} \otimes \mathbb{1}\right)(\rho)\right] .
$$

Clearly, $\mathfrak{F}_{N} \geq N \mathfrak{F}_{1}$ using the additivity of the QFI. An upper bound on $\mathfrak{F}_{N}\left(\mathcal{E}_{\omega}\right)$ could be derived from Eq. (6) $[24,28]$ (see also Appendix A),

$$
\mathfrak{F}_{N}\left(\mathcal{E}_{\omega}\right) \leq 4 \min _{h}\left[N\|\alpha\|+N(N-1)\|\beta\|^{2}\right],
$$

where $\beta=i \mathbf{K}^{\dagger}(\dot{\mathbf{K}}-i h \mathbf{K})$. If there is an $h$ such that $\beta=0$,

$$
\mathfrak{F}_{N}\left(\mathcal{E}_{\omega}\right) \leq 4 \min _{h: \beta=0} N\|\alpha\|
$$

and $\mathfrak{F}_{N}\left(\mathcal{E}_{\omega}\right)$ follows the SQL asymptotically.
The metrological protocols we considered in Fig. 1(b) are usually called parallel strategies where $N$ identical quantum channels act in parallel on a quantum state [31]. Researchers also consider sequential strategies where we allow quantum controls (arbitrary quantum operations) between each quantum channel [see Fig. 1(c)]. The QFI optimized over all possible inputs and quantum controls has the upper bound [31,32]

$$
\begin{aligned}
\mathfrak{F}_{N}^{\text {(seq) }}\left(\mathcal{E}_{\omega}\right) \leq & 4 \min _{h}[N\|\alpha\|+N(N-1)\|\beta\|(\|\beta\| \\
& +2 \sqrt{\|\alpha\|})] .
\end{aligned}
$$

Therefore, for all $h, \beta \neq 0$ is also a necessary condition to achieve the HL for sequential strategies. When the condition is violated, there exists an $h$ such that $\beta=0$ and $\mathfrak{F}_{N}^{(\text {seq) }}\left(\mathcal{E}_{\omega}\right)$ has the same upper bound [Eq. (9)] as $\mathfrak{F}_{N}\left(\mathcal{E}_{\omega}\right)$. Sequential strategies are more powerful than parallel strategies because they can simulate parallel strategies using the same input states and swap operators as quantum controls [31].

\section{B. Main results}

In fact, the condition that, for all $h, \beta \neq 0$ is equivalent to $H \notin \mathcal{S}$, where

$$
H=i \mathbf{K}^{\dagger} \dot{\mathbf{K}}, \quad \mathcal{S}=\operatorname{span}_{\mathbb{H}}\left\{K_{i}^{\dagger} K_{j} \text { for all } i, j\right\} .
$$

Here $\operatorname{span}_{\mathbb{H}}\{\cdot\}$ represents all Hermitian operators that are linear combinations of operators in $\{\cdot\}$. We call it the "Hamiltonian-not-in-Kraus-span" (HNKS) condition. One can check that $H$ and $\beta$ are always Hermitian by taking the derivative of $\mathbf{K}^{\dagger} \mathbf{K}=I$. Note that different Kraus representations may lead to different $H$, but they only differ by some operator inside $\mathcal{S}$, so whether $H \in \mathcal{S}$ or $H \notin \mathcal{S}$ does not depend on the choice of Kraus representation. For a unitary channel $r=1$ and $K_{1}=U_{\omega}=e^{-i H \omega}, H=$ $i U_{\omega}^{\dagger} \dot{U}_{\omega}$ is exactly the Hamiltonian for $\omega$, explaining its name. The HL is achievable for unitary channels because $\mathcal{S}=\operatorname{span}_{\mathbb{H}}\{I\}$ and we always have $H \notin \mathcal{S}$ for nontrivial $H$. It is only possible to achievable the HL if the HNKS condition holds.

We show in Sec. V that the HNKS condition is also a sufficient condition to achieve the HL for parallel strategies in Fig. 1(b), and, hence, sequential strategies in Fig. 1(c) that contain the former. We summarize this in the following theorem.

Theorem 1. We have $\mathfrak{F}_{N}\left(\mathcal{E}_{\omega}\right)=\Theta\left(N^{2}\right)$ if and only if $H \notin$ $\mathcal{S}$. Otherwise, $\mathfrak{F}_{N}\left(\mathcal{E}_{\omega}\right)=\Theta(N)$. The statement is also true for $\mathfrak{F}_{N}^{\text {(seq) }}\left(\mathcal{E}_{\omega}\right)$.

Furthermore, the QFI upper bound in Eq. (9) is achievable asymptotically when $H \in \mathcal{S}$ for both parallel and sequential strategies. 
Theorem 2. When $H \in \mathcal{S}$,

$$
\mathfrak{F}_{\mathrm{SQL}}\left(\mathcal{E}_{\omega}\right):=\lim _{N \rightarrow \infty} \mathfrak{F}_{N}\left(\mathcal{E}_{\omega}\right) / N=4 \min _{h: \beta=0}\|\alpha\| .
$$

For any $\eta>0$, there exists an input state $\left|\psi_{\eta, N}\right\rangle$ computable via a SDP such that $\lim _{N \rightarrow \infty} F\left(\left(\mathcal{E}_{\omega}^{\otimes N} \otimes\right.\right.$ $\left.\mathbb{1})\left(\left|\psi_{\eta, N}\right\rangle\right)\right) / N>\mathfrak{F}_{\mathrm{SQL}}\left(\mathcal{E}_{\omega}\right)-\eta$. Furthermore, $\mathfrak{F}_{\mathrm{SQL}}^{(\mathrm{seq})}\left(\mathcal{E}_{\omega}\right)$ $=\mathfrak{F}_{\mathrm{SQL}}\left(\mathcal{E}_{\omega}\right)$.

Note that $\mathfrak{F}_{\mathrm{SQL}}\left(\mathcal{E}_{\omega}\right)$ is called the "asymptotic channel QFI" in Ref. [29]. The quadratic term of the QFI upper bound in Eq. (8) is also achievable when $H \notin \mathcal{S}$ for parallel strategies.

Theorem 3. When $H \notin \mathcal{S}$,

$$
\mathfrak{F}_{\mathrm{HL}}\left(\mathcal{E}_{\omega}\right):=\lim _{N \rightarrow \infty} \mathfrak{F}_{N}\left(\mathcal{E}_{\omega}\right) / N^{2}=4 \min _{h}\|\beta\|^{2} .
$$

There exists an input state $\left|\psi_{N}\right\rangle$ computable via a SDP such that $F\left(\left(\mathcal{E}_{\omega}^{\otimes N} \otimes \mathbb{1}\right)\left(\left|\psi_{N}\right\rangle\right)\right) / N^{2}=\mathfrak{F}_{\mathrm{HL}}\left(\mathcal{E}_{\omega}\right)$.

Note that, without the help of the ancilla system, the QFI upper bound in Eq. (9) may not be achievable asymptotically $[30,71]$. For example, the upper bound in Eq. (9) for phase estimation in amplitude damping channels is reduced by a factor of 4 without an ancilla [30,31].

Although the theorems above for general quantum channel estimation have not been proven prior to this work, we note here that in the special case of Hamiltonian estimation under Markovian noise with the assistance of fast and frequency quantum controls, i.e., sequential strategies [Fig. 1(c)] where $\mathcal{E}_{\omega, d t}(\rho)=-i\left[\omega H^{\prime}, \rho\right] d t+\sum_{i}\left(L_{i} \rho L_{i}^{\dagger}-\right.$ $\left.\frac{1}{2}\left\{L_{i}^{\dagger} L_{i}, \rho\right\}\right)+O\left(d t^{2}\right)$, with $t$ the probing time and $N=$ $t / d t$, the results (when taking the limit $d t \rightarrow 0$ ) are already known. The necessity part was proven in Refs. [32-34] and the sufficiency part was proven in Refs [34,52]. In particular, it was shown that in this case, the HNKS condition reduces to the condition $H^{\prime} \notin \mathcal{S}^{\prime}$, where $\mathcal{S}^{\prime}=$ $\operatorname{span}_{\mathbb{H}}\left\{I, L_{i}, L_{i}^{\dagger}, L_{i}^{\dagger} L_{j}\right.$, for all $\left.i, j\right\}$ is called the Lindblad span. The condition was first named the "Hamiltonian-notin-Lindblad-span" condition in Ref. [34].

Theorem 2 indicates that, when the HNKS condition is violated (which almost surely happens statistically), there is no advantage of sequential strategies over parallel strategies asymptotically, as conjectured in Ref. [31]. Interestingly, similar results were discovered for quantum channel discrimination, a related field [35,36,83-87]. It was recently proven that sequential strategies cannot outperform parallel strategies asymptotically in asymmetric discrimination of two arbitrary quantum channels [88-92]. Our result is different, however, because the QFI cannot be characterized as the limit of quantum relative entropy [83] and it is also unclear how to interpret the (a)

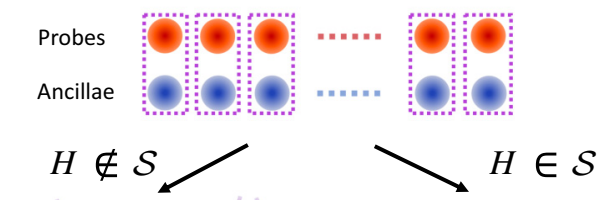

(b)

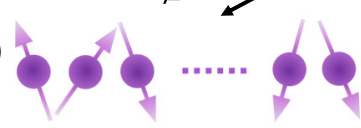

Noiseless logical qubits

(c)

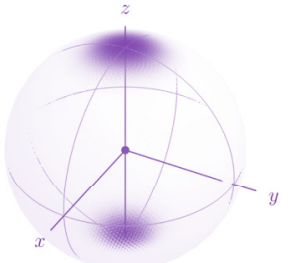

Logical GHZ state (d)

Noisy logical qubits

(e)

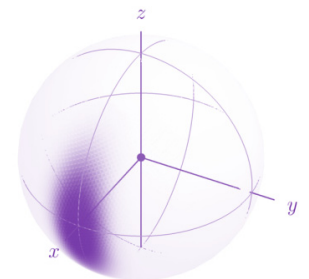

Logical spin-squeezed state

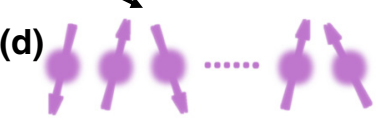

FIG. 2. The optimal metrological protocol. (a) The original physical system where we have $N$ noisy probes and $N$ noiseless ancillae. Each pair of probe-ancilla subsystem (purple box) encodes a logical qubit (see Sec. IV). (b),(c) When $H \notin \mathcal{S}$, the logical qubits are noiseless. We choose the GHZ state of $N$-logical qubits as the optimal input. (d),(e) When $H \in S$, each logical qubit is subject to an effective dephasing noise. We choose the spin-squeezed state of the $N$-logical qubits with suitable parameters as the optimal input. We plot the quasiprobability distribution $Q(\theta, \varphi)=|\langle\theta, \varphi \mid \psi\rangle|^{2}$ on a sphere using coordinates $(x, y, z)=(\sin \theta \cos \varphi, \sin \theta \sin \varphi, \cos \theta)$ [94], where $|\theta, \varphi\rangle=\left[\cos (\theta / 2)|0\rangle+e^{i \varphi} \sin (\theta / 2)|1\rangle\right]^{\otimes N}$ and $N=50$. (Darker colors indicate larger values.)

HNKS condition in terms of asymmetric channel discrimination. Moreover, we provide a constructive proof with explicit and efficiently computable QEC metrological protocols, which paves the way for practical implementation of error-corrected sensing schemes.

Based on the previous discussion, in order to prove the theorems, it is sufficient to provide a QEC protocol using parallel strategies that achieve the QFI upper bound [Eq. (8)] asymptotically when $H \in \mathcal{S}$ or $H \notin \mathcal{S}$. Thus, we focus only on parallel strategies in the following. We first show that Theorems 3 and 2 hold for the generalized single-qubit dephasing channels in Sec. III where both the phase and the noise parameter vary with respect to $\omega$. Then we generalized the results to arbitrary quantum channels $\mathcal{E}_{\omega}$ using a QEC protocol in Secs. IV and VI. The two steps are summarized in Fig. 2.

\section{SINGLE-QUBIT DEPHASING CHANNELS}

According to Eq. (8), $\mathfrak{F}_{\mathrm{HL}} \leq \mathfrak{F}_{\mathrm{HL}}^{(u)}$ and $\mathfrak{F}_{\mathrm{SQL}} \leq \mathfrak{F}_{\mathrm{SQL}}^{(u)}$, where $\mathfrak{F}_{\mathrm{HL}}^{(u)}:=4 \min _{h}\|\beta\|^{2}$ and $\mathfrak{F}_{\mathrm{SQL}}^{(u)}:=4 \min _{h: \beta=0}\|\alpha\|$. The superscript " $(u)$ " refers to the upper bounds here. In this section, we show that the above equalities hold for any 
single-qubit dephasing channel

$$
\mathcal{D}_{\omega}(\rho)=(1-p) e^{-i \phi \sigma_{z} / 2} \rho e^{i \phi \sigma_{z} / 2}+p \sigma_{z} e^{-i \phi \sigma_{z} / 2} \rho e^{i \phi \sigma_{z} / 2} \sigma_{z},
$$

which is the composition of the conventional dephasing channel $\rho \mapsto(1-p) \rho+p \sigma_{z} \rho \sigma_{z}(0 \leq p<1)$ and the rotation in the $z$ direction $\rho \mapsto e^{-i \phi \sigma_{z} / 2} \rho e^{i \phi \sigma_{z} / 2}$. Both $p$ and $\phi$ are functions of an unknown parameter $\omega$. As shown in Appendix B, the HNKS condition is equivalent to $p=0$ and the QFI upper bounds for $\mathcal{D}_{\omega}$ are

$$
\mathfrak{F}_{\mathrm{HL}}^{(u)}\left(\mathcal{D}_{\omega}\right)=|\dot{\xi}|^{2}, \quad \mathfrak{F}_{\mathrm{SQL}}^{(u)}\left(\mathcal{D}_{\omega}\right)=\frac{|\dot{\xi}|^{2}}{1-|\xi|^{2}}
$$

where $\xi=\left\langle 0\left|\mathcal{D}_{\omega}(|0\rangle\langle 1|)\right| 1\right\rangle=(1-2 p) e^{-i \phi}$.

Now we show that $\mathfrak{F}_{\mathrm{HL}, \mathrm{SQL}}\left(\mathcal{D}_{\omega}\right)=\mathfrak{F}_{\mathrm{HL}, \mathrm{SQL}}^{(u)}\left(\mathcal{D}_{\omega}\right)$ and provide the optimal input states in both cases. When the HNKS condition is satisfied $(p=0), \mathcal{D}_{\omega}$ is unitary. Using the GHZ state $\left|\psi_{0}\right\rangle=(1 / \sqrt{2})\left(|0\rangle^{\otimes N}+|1\rangle^{\otimes N}\right)$ as the input state, we could achieve

$$
F\left(\mathcal{D}_{\omega}^{\otimes N}\left(\left|\psi_{0}\right\rangle\left\langle\psi_{0}\right|\right)\right)=|\dot{\xi}|^{2} N^{2},
$$

which implies that $\mathfrak{F}_{\mathrm{HL}}\left(\mathcal{D}_{\omega}\right)=\mathfrak{F}_{\mathrm{HL}}^{(u)}\left(\mathcal{D}_{\omega}\right)$.

To calculate the optimal QFI when the HNKS condition is violated $(p>0)$, we use the following two useful formulae. For any pure state input $\left|\psi_{0}\right\rangle$ and output $\rho_{\omega}=$ $\mathcal{D}_{\omega}^{\otimes N}\left(\left|\psi_{0}\right\rangle\left\langle\psi_{0}\right|\right)$, we have, for all $N$,

$$
F\left(\rho_{\omega}\right)=F_{p}\left(\rho_{\omega}\right)+F_{\phi}\left(\rho_{\omega}\right),
$$

where $F_{p}\left(\rho_{\omega}\right)=\operatorname{Tr}\left(L_{p}^{2} \rho_{\omega}\right)$ is the QFI with respect to $\omega$ when only the noise parameter $p$ varies with respect to $\omega$, where the SLD $L_{p}$ satisfies

$$
\frac{\partial \rho_{\omega}}{\partial p} \dot{p}=\frac{1}{2} L_{p} \rho_{\omega}+\rho_{\omega} L_{p} .
$$

Similarly, $F_{\phi}\left(\rho_{\omega}\right)$ is the QFI with respect to $\omega$ when only the phase parameter $\phi$ varies with respect to $\omega$. The proof of Eq. (17) is provided in Appendix C. Another useful formula is the error propagation formula [93]

$$
F(\rho) \geq \frac{1}{\left\langle\Delta J^{2}\right\rangle_{\rho}}\left(\frac{\partial\langle J\rangle_{\rho}}{\partial \omega}\right)^{2}
$$

for arbitrary $\rho$ as a function of $\omega$ and arbitrary Hermitian operator $J$, where $\langle J\rangle_{\rho}=\operatorname{Tr}(J \rho)$ and $\left\langle\Delta J^{2}\right\rangle_{\rho}=\left\langle J^{2}\right\rangle_{\rho}-$ $\langle J\rangle_{\rho}^{2}$. The equality holds when $J$ is equal to the SLD operator of $\rho$.

Consider an $N$-qubit spin-squeezed state $[39,94]$

$$
\left|\psi_{\mu, \nu}\right\rangle=e^{-i \nu J_{x}} e^{-i \mu J_{z}^{2} / 2} e^{-i \pi J_{y} / 2}|0\rangle^{\otimes N},
$$

where $J_{x, y, z}=\frac{1}{2} \sum_{k=1}^{N} \sigma_{x, y, z}^{(k)}$ with the superscript “ $(k)$ " denoting operators on the $k$ th qubit. Let $\left|\psi_{0}\right\rangle=$
TABLE I. Comparison between Sec. III and previous works.

\begin{tabular}{lcc}
\hline \hline Case & Channel & Attainability of Eq. (15) \\
\hline$H \notin \mathcal{S}(p=0)$ & $\mathcal{D}_{\omega}=\mathcal{U}_{\omega}$ & Attainable (see, e.g., Ref. [23]) \\
$H \in \mathcal{S}(p \neq 0)$ & $\mathcal{D}_{\omega}$ when $\dot{p}=0$ & Attainable (see, e.g., Ref. [39]) \\
$H \in \mathcal{S}(p \neq 0)$ & $\mathcal{D}_{\omega}$ when $\dot{\phi}=0$ & Attainable (see, e.g., Ref. [95]) \\
$H \in \mathcal{S}(p \neq 0)$ & General $\mathcal{D}_{\omega}$ & Unknown until this work \\
\hline \hline
\end{tabular}

$e^{i \phi J_{z}}\left|\psi_{\mu, \nu}\right\rangle$. Using Eqs. (17) and (19), we have, for $\rho_{\omega}=$ $\mathcal{D}_{\omega}^{\otimes N}\left(\left|\psi_{0}\right\rangle\left\langle\psi_{0}\right|\right)$,

$$
\begin{aligned}
F\left(\rho_{\omega}\right) \geq & \frac{1}{\left\langle\Delta J_{x}^{2}\right\rangle_{\rho_{\omega}}}\left(\frac{\partial\left\langle J_{x}\right\rangle_{\rho_{\omega}}}{\partial p} \dot{p}\right)^{2} \\
& +\frac{1}{\left\langle\Delta J_{y}^{2}\right\rangle}\left(\frac{\partial\left\langle J_{y}\right\rangle_{\rho_{\omega}}}{\partial \phi} \dot{\phi}\right)^{2} .
\end{aligned}
$$

As shown in Appendix $\mathrm{D}$, as $N \rightarrow \infty$, with suitable choices of $(\mu, v)$, we have (up to the lowest order of N) $\left\langle\Delta J_{x}^{2}\right\rangle_{\rho_{\omega}} \approx\left\langle\Delta J_{y}\right\rangle_{\rho_{\omega}}^{2} \approx p(1-p) N,\left(\partial\left\langle J_{x}\right\rangle_{\rho_{\omega}} / \partial p\right) \dot{p} \approx$ $-\dot{p} N$, and $\left(\partial\left\langle J_{y}\right\rangle_{\rho_{\omega}} / \partial \phi\right) \dot{\phi} \approx(1-2 p) \dot{\phi} N / 2$. For example, we can choose

$$
\begin{aligned}
\mu & =4\left(\frac{2}{N}\right)^{5 / 6} \text { and } \\
\nu & =\frac{\pi}{2}-\frac{1}{2} \arctan \frac{4 \sin (\mu / 2) \cos ^{N-2}(\mu / 2)}{1-\cos ^{N-2} \mu} .
\end{aligned}
$$

The corresponding $\left|\psi_{\mu, \nu}\right\rangle$ is illustrated in Fig. 2(e) using the quasiprobability distribution $Q(\theta, \varphi)=$ $\left|\left\langle\theta, \varphi \mid \psi_{\mu, \nu}\right\rangle\right|^{2}$ on a sphere [94]. Therefore,

$$
F\left(\rho_{\omega}\right) \geq \frac{|\dot{\xi}|^{2}}{1-|\xi|^{2}} N+o(N)
$$

which implies that $\mathfrak{F}_{\mathrm{SQL}}\left(\mathcal{D}_{\omega}\right)=\mathfrak{F}_{\mathrm{SQL}}^{(u)}\left(\mathcal{D}_{\omega}\right)$. Compared with $\mathfrak{F}_{1}\left(\mathcal{D}_{\omega}\right)$ (see Appendix B), $\mathfrak{F}_{\mathrm{SQL}}\left(\mathcal{D}_{\omega}\right)$ has a factor of $1 /[4 p(1-p)]$ enhancement when we estimate the phase parameter $(\dot{p}=0)$. When we estimate the noise parameter $(\dot{\phi}=0)$, however, $\mathfrak{F}_{\mathrm{SQL}}\left(\mathcal{D}_{\omega}\right)=\mathfrak{F}_{1}\left(\mathcal{D}_{\omega}\right)$. In general, $\mathfrak{F}_{\mathrm{SQL}} / \mathfrak{F}_{1}$ is between 1 and $1 /[4 p(1-p)]$.

In summary, Theorems 2 and 3 hold for dephasing channels. We also compare our results in this section with the known results in Table I. The ancilla is not required here. When the noise is nonzero, the QFI must follow the SQL and there exists a spin-squeezed state achieving the QFI asymptotically. In particular, the squeezing parameter should be tuned carefully such that both the $J_{x}$ and $J_{y}$ variance are small such that both the noise and the phase parameter are estimated with optimal precision.

\section{THE QEC PROTOCOL}

In this section, we introduce a QEC protocol such that every quantum channel simulates the dephasing channel 
introduced in Sec. III. To be specific, we find the encoding channel $\mathcal{E}_{\text {enc }}$ and the recovery channel $\mathcal{R}$ such that

$$
\mathcal{R} \circ \mathcal{E}_{\omega} \circ \mathcal{E}_{\mathrm{enc}}=\mathcal{D}_{L, \omega}
$$

The construction fully utilizes the advantage of the ancilla, which has the same dimension as the probe with an extra qubit. Let $\operatorname{dim} \mathcal{H}_{\mathcal{P}}=d$ and $\operatorname{dim} \mathcal{H}_{\mathcal{A}}=2 d$. We pick a QEC code

$$
\left|0_{L}\right\rangle=\sum_{i, j=1}^{d} A_{0, i j}|i\rangle_{\mathcal{P}}|j, 0\rangle_{\mathcal{A}}, \quad\left|1_{L}\right\rangle=\sum_{i, j=1}^{d} A_{1, i j}|i\rangle_{\mathcal{P}}|j, 1\rangle_{\mathcal{A}},
$$

with encoding channel $\mathcal{E}_{\text {enc }}(\cdot)=V(\cdot) V^{\dagger}$, where $V=$ $\left|0_{L}\right\rangle\left\langle 0|+| 1_{L}\right\rangle\langle 1|$, and a recovery channel

$$
\begin{aligned}
\mathcal{R}(\cdot)=\sum_{m=1}^{M}\left(|0\rangle\left\langle R_{m}, 0|+| 1\right\rangle\left\langle Q_{m}, 1\right|\right)(\cdot) \\
\quad \times\left(\left|R_{m}, 0\right\rangle\left\langle 0|+| Q_{m}, 1\right\rangle\langle 1|\right) .
\end{aligned}
$$

Here $A_{0,1}$ are matrices in $\mathbb{C}^{d \times d}$ satisfying $\operatorname{Tr}\left(A_{0,1}^{\dagger} A_{0,1}\right)=$ 1 , and $R=\left(\left|R_{1}\right\rangle \cdots\left|R_{M}\right\rangle\right)$ and $Q=\left(\left|Q_{1}\right\rangle \cdots\left|Q_{M}\right\rangle\right)$ are matrices satisfying $R R^{\dagger}=Q Q^{\dagger}=I$. The last ancillary qubit in $\mathcal{H}_{\mathcal{A}}$ guarantees the logical channel to be dephasing, which satisfies

$$
\xi=\sum_{i, m}\left\langle R_{m}, 0\left|K_{i}\right| 0_{L}\right\rangle\left\langle 1_{L}\left|K_{i}^{\dagger}\right| Q_{m}, 1\right\rangle,
$$

and $\mathfrak{F}_{\mathrm{HL}, \mathrm{SQL}}\left(\mathcal{D}_{L, \omega}\right)$ could then be directly calculated using Eq. (15). Note that in the equation above and in what follows we use $K_{i}$ as a substitute for $K_{i} \otimes I$ to ease notation. Below, we show that, by optimizing $\mathfrak{F}_{\mathrm{HL}, \mathrm{SQL}}\left(\mathcal{D}_{L, \omega}\right)$ over both the recovery channel $(R, Q)$ and the QEC code $\left(A_{0,1}\right)$, the QFI upper bounds $\mathfrak{F}_{\mathrm{HL}, \mathrm{SQL}}^{(u)}\left(\mathcal{E}_{\omega}\right)$ are achievable.

\section{ACHIEVING THE HL UPPER BOUND}

When $H \notin \mathcal{S}$, we construct a QEC code such that the HL upper bound $\mathfrak{F}_{\mathrm{HL}}^{(u)}\left(\mathcal{E}_{\omega}\right)$ is achieved. For dephasing channels, the HL is achievable only if $|\xi|=1$. Since any transformation $R \leftarrow e^{i \varphi} R$ does not affect the QFI, without loss of generality, we assume that $\xi=1$. This means that the QEC has to be perfect, i.e., satisfies the Knill-Laflamme condition [60]

$$
P K_{i}^{\dagger} K_{j} P \propto P \quad \text { for all } i, j,
$$

where $P=\left|0_{L}\right\rangle\left\langle 0_{L}|+| 1_{L}\right\rangle\left\langle 1_{L}\right|$. Moreover, there exists a Kraus representation $\left\{K_{i}^{\prime}\right\}_{i=1}^{r^{\prime}}$ such that $P K_{i}^{\prime \dagger} K_{j}^{\prime} P=\mu_{i} \delta_{i j} P$ and $K_{i}^{\prime} P=U_{i} \sqrt{\mu_{i}} P$. The unitary $U_{i}$ has the form

$$
U_{i}=U_{0, i} \otimes|0\rangle\left\langle 0\left|+U_{1, i} \otimes\right| 1\right\rangle\langle 1|,
$$

where $U_{0, i}$ and $U_{1, i}$ are also unitary. Let

$$
\left|R_{i}\right\rangle=\left\langle 0\left|U_{i}\right| 0_{L}\right\rangle, \quad\left|Q_{i}\right\rangle=\left\langle 0\left|U_{i}\right| 0_{L}\right\rangle,
$$

for $1 \leq i \leq r^{\prime}$. We could also add some additional $\left|R_{i}\right\rangle$ and $\left|Q_{i}\right\rangle$ to them to make sure that they are two complete and orthonormal bases. Then one could verify that $\xi=1$ and

$$
\dot{\xi}=-i \operatorname{Tr}\left[(H \otimes I) \sigma_{z, L}\right],
$$

where $\sigma_{z, L}=\left|0_{L}\right\rangle\left\langle 0_{L}|-| 1_{L}\right\rangle\left\langle 1_{L}\right|$. Let $\tilde{C}=A_{0} A_{0}^{\dagger}-A_{1} A_{1}^{\dagger}$, $\dot{\xi}=-i \operatorname{Tr}(H \tilde{C})$. Then the Knill-Laflamme condition is equivalent to $\operatorname{Tr}(\tilde{C} S)=0$ for all $S \in \mathcal{S}$. The optimization of the QFI over the QEC code becomes

$$
\operatorname{maximize}|\dot{\xi}|=|\operatorname{Tr}(H \tilde{C})|
$$

$$
\begin{aligned}
& \text { subject to }\|\tilde{C}\|_{1} \leq 2, \quad \operatorname{Tr}(\tilde{C} S)=0 \\
& \text { for all } \tilde{C} \in \mathbb{H}_{d}, \quad S \in \mathcal{S},
\end{aligned}
$$

where $\|\cdot\|_{1}$ is the trace norm. A similar SDP problem was considered in Ref. [34]. The optimal $|\dot{\xi}|$ is equal to $2 \min _{S \in \mathcal{S}}\|H-S\|$ and the optimal $\tilde{C}$ could be found via a SDP. Any $A_{0}, A_{1}$ such that $\tilde{C}$ is optimal would achieve the optimal QFI. This means that there exists an encoding, and therefore an optimal input state $\left|\psi_{N}\right\rangle$ that is the logical GHZ state such that

$$
\lim _{N \rightarrow \infty} \frac{F\left(\left(\mathcal{E}_{\omega}^{\otimes N} \otimes \mathbb{1}\right)\left(\left|\psi_{N}\right\rangle\right)\right)}{N^{2}}=4 \min _{S \in \mathcal{S}}\|H-S\|^{2} .
$$

Clearly, $\quad 4 \min _{S \in \mathcal{S}}\|H-S\|^{2}=4 \min _{h}\|\beta\|^{2}=\mathfrak{F}_{\mathrm{HL}}^{(u)}\left(\mathcal{E}_{\omega}\right)$, where we have used the fact that, for any $S \in \mathcal{S}$, there exists an $h \in \mathbb{H}_{r}$ such that $S=\mathbf{K}^{\dagger} h \mathbf{K}$ and vice versa. Theorem 3 is then proven. Note that, given the optimal $\tilde{C}$, we can always choose $A_{0} A_{0}^{\dagger}$ and $A_{1} A_{1}^{\dagger}$ with orthogonal supports and the last ancillary qubit in $\mathcal{H}_{\mathcal{A}}$ could be removed because $\left|0_{L}\right\rangle$ and $\left|1_{L}\right\rangle$ in this case could be distinguished using projections onto the orthogonal supports in $\mathcal{H}_{\mathcal{A}}$ [34]. Therefore, a $d$-dimensional ancillary system is sufficient.

We have demonstrated the QEC code achieving the optimal HL for arbitrary quantum channels. The code is designed to satisfy the Knill-Laflamme condition and optimize the QFI. The logical dephasing channel is exactly the identity channel at the true value of $\omega$ and any change in $\omega$ results in a detectable phase, allowing it to be estimated at the HL. 


\section{ACHIEVING THE SQL UPPER BOUND}

When $H \in \mathcal{S}$, the situation is much more complicated because, when $|\xi|=1$, we must also have $|\dot{\xi}|=0$ and no signal can be detected. Therefore, we must consider the trade-off between maximizing the signal and minimizing the noise. To be exact, we want to maximize

$$
\mathfrak{F}_{\mathrm{SQL}}\left(\mathcal{D}_{L, \omega}\right)=\frac{|\dot{\xi}|^{2}}{1-|\xi|^{2}} .
$$

We show, for any $\eta>0$, there exists a near-optimal code and recovery such that $\mathfrak{F}_{\mathrm{SQL}}\left(\mathcal{D}_{L, \omega}\right)>\mathfrak{F}_{\mathrm{SQL}}^{(u)}\left(\mathcal{E}_{\omega}\right)-\eta$, proving Theorem 2 . We only consider the case where $\mathfrak{F}_{\mathrm{SQL}}\left(\mathcal{E}_{\omega}\right)>\mathfrak{F}_{1}\left(\mathcal{E}_{\omega}\right)>0$ because otherwise $\mathfrak{F}_{1}\left(\mathcal{E}_{\omega}\right)=$ $\mathfrak{F}_{\mathrm{SQL}}\left(\mathcal{E}_{\omega}\right)$ and product states are sufficient to achieve $\mathfrak{F}_{\mathrm{SQL}}\left(\mathcal{E}_{\omega}\right)$. Detailed derivations can be found in Appendix $\mathrm{E}$; we provide a sketch of the proof here. To simplify the calculation, we consider a special type of code, the perturbation code, first introduced in Ref. [52], where

$$
A_{0}=\sqrt{1-\varepsilon^{2}} C+\varepsilon D, \quad A_{1}=\sqrt{1-\varepsilon^{2}} C-\varepsilon D,
$$

satisfying $\operatorname{Tr}\left(C^{\dagger} D\right)=0$ and $\operatorname{Tr}\left(C^{\dagger} C\right)=\operatorname{Tr}\left(D^{\dagger} D\right)=1$. In this section, we define $\tilde{C}=C D^{\dagger}+D C^{\dagger}$ (differing by a factor of $\varepsilon \sqrt{1-\varepsilon^{2}}$ from the $\tilde{C}$ defined in Sec. V) and also assume that $C$ is full rank so that $\tilde{C}$ can be an arbitrary Hermitian matrix. Here $\varepsilon$ is a small constant and we calculate $\mathfrak{F}_{\mathrm{SQL}}\left(\mathcal{D}_{L, \omega}\right)$ up to the lowest order of $\varepsilon$. We adopt the small $\varepsilon$ treatment because it allows us to mathematically simplify the optimization of Eq. (34), though it is surprising that the optimal QFI is achievable in such a regime where both the signal and the noise are small. Heuristically, it comes from an observation that sometimes the absolute strengths of the signal and the noise are not important - they could cancel each other out in the numerator and the denominator and only the ratio between them matters. See Appendix G of Ref. [52] for an example.

To proceed, we first introduce the vectorization of matrices $|\star\rangle\rangle=\sum_{i j} \star_{i j}|i\rangle|j\rangle$ for all $\star \in \mathbb{C}^{d \times d}$ to simplify the notation. We define $E_{0,1}, E, F \in \mathbb{C}^{d^{2} \times r}$ as

$$
\begin{gathered}
\left.\left.E_{0,1}=\left(\left|K_{1} A_{0,1}\right\rangle\right\rangle \cdots\left|K_{r} A_{0,1}\right\rangle\right\rangle\right), \\
\left.\left.\left.\left.E=\left(\left|K_{1} C\right\rangle\right\rangle \cdots\left|K_{r} C\right\rangle\right\rangle\right), \quad F=\left(\left|K_{1} D\right\rangle\right\rangle \cdots\left|K_{r} D\right\rangle\right\rangle\right),
\end{gathered}
$$

which satisfy $E_{0,1}=\sqrt{1-\varepsilon^{2}} \pm \varepsilon F, \operatorname{Tr}\left(E^{\dagger} F\right)=0$, and $\operatorname{Tr}\left(E^{\dagger} E\right)=\operatorname{Tr}\left(F^{\dagger} F\right)=1$. Let the recovery matrix $T=$ $Q R^{\dagger} \in \mathbb{C}^{d^{2} \times d^{2}}$; then

$$
\xi=\operatorname{Tr}\left(T E_{0} E_{1}^{\dagger}\right), \quad \dot{\xi}=\operatorname{Tr}\left(T \dot{E}_{0} E_{1}^{\dagger}\right)+\operatorname{Tr}\left(T E_{0} \dot{E}_{1}^{\dagger}\right) .
$$

We consider the regime where both the signal and the noise are sufficiently small — both the denominator and the numerator in Eq. (34) will be $O\left(\varepsilon^{2}\right)$. The recovery matrix $T$ should also be close to the identity operator. We assume that $T=e^{i \varepsilon G}$, where $G$ is Hermitian, and let $\sigma=E E^{\dagger}$, $\tilde{\sigma}=i\left(F E^{\dagger}-E F^{\dagger}\right)$. Expanding $T, E_{0}, E_{1}$ around $\varepsilon=0$, we first optimize $\mathfrak{F}_{\mathrm{SQL}}\left(\mathcal{D}_{L, \omega}\right)$ over all possible $G$, i.e.,

$\mathfrak{F}_{\mathrm{SQL}}\left(\mathcal{D}_{L, \omega}\right) \approx \max _{G} \frac{|\operatorname{Tr}(G \dot{\sigma})|^{2}}{4-2 \operatorname{Tr}(G \tilde{\sigma})+\operatorname{Tr}\left(G^{2} \sigma\right)-|\operatorname{Tr}(G \sigma)|^{2}}$,

up to the lowest order of $\varepsilon$. The maximization can be calculated by taking the derivative with respect to $G$. We can show that the optimal $G$ is

$$
G_{\mathrm{opt}}=\frac{\left[4-\operatorname{Tr}\left(L_{\sigma}[\tilde{\sigma}] \tilde{\sigma}\right)\right]}{\operatorname{Tr}\left(L_{\sigma}[\dot{\sigma}] \tilde{\sigma}\right)} L_{\sigma}[\dot{\sigma}]+L_{\sigma}[\tilde{\sigma}]
$$

and that the corresponding optimal QFI is

$$
\mathfrak{F}_{\mathrm{SQL}}\left(\mathcal{D}_{L, \omega}\right) \approx \operatorname{Tr}\left(L_{\sigma}[\dot{\sigma}] \dot{\sigma}\right)+\frac{\operatorname{Tr}\left(L_{\sigma}[\dot{\sigma}] \tilde{\sigma}\right)^{2}}{4-\operatorname{Tr}\left(L_{\sigma}[\tilde{\sigma}] \tilde{\sigma}\right)}
$$

Now $\mathfrak{F}_{\mathrm{SQL}}\left(\mathcal{D}_{L, \omega}\right)$ is a function of the code $(C$ and $D)$ only. We further simplify by writing it as a function of only $C$ and $\tilde{C}$. Let $\tau=E^{\dagger} E, \tilde{\tau}=E^{\dagger} F+F^{\dagger} E, \tau^{\prime}=i E^{\dagger} \dot{E}-i \dot{E}^{\dagger} E$ such that

$$
\begin{gathered}
\tau_{i j}=\operatorname{Tr}\left(C^{\dagger} K_{i}^{\dagger} K_{j} C\right), \quad \tilde{\tau}_{i j}=\operatorname{Tr}\left(\tilde{C} K_{i}^{\dagger} K_{j}\right), \\
\tau_{i j}^{\prime}=i \operatorname{Tr}\left(C^{\dagger} K_{i}^{\dagger} \dot{K}_{j} C\right)-i \operatorname{Tr}\left(C^{\dagger} \dot{K}_{i}^{\dagger} K_{j} C\right) .
\end{gathered}
$$

Then we can verify that

$$
\begin{gathered}
\operatorname{Tr}\left(L_{\sigma}[\dot{\sigma}] \dot{\sigma}\right)=4 \operatorname{Tr}\left(C^{\dagger} \dot{\mathbf{K}}^{\dagger} \dot{\mathbf{K}} C\right)-\operatorname{Tr}\left(L_{\tau}\left[\tau^{\prime}\right] \tau^{\prime}\right), \\
\operatorname{Tr}\left(L_{\sigma}[\dot{\sigma}] \tilde{\sigma}\right)=-2 \operatorname{Tr}(\tilde{C} H)+\operatorname{Tr}\left(L_{\tau}\left[\tau^{\prime}\right] \tilde{\tau}\right), \\
\operatorname{Tr}\left(L_{\sigma}[\tilde{\sigma}] \tilde{\sigma}\right)=4-\operatorname{Tr}\left(L_{\tau}[\tilde{\tau}] \tilde{\tau}\right),
\end{gathered}
$$

and

$$
\begin{aligned}
\mathfrak{F}_{\mathrm{SQL}}\left(\mathcal{D}_{L, \omega}\right) \approx & f(C, \tilde{C}) \\
= & 4 \operatorname{Tr}\left(C^{\dagger} \dot{\mathbf{K}}^{\dagger} \dot{\mathbf{K}} C\right)-\operatorname{Tr}\left(L_{\tau}\left[\tau^{\prime}\right] \tau^{\prime}\right) \\
& +\frac{\left[-2 \operatorname{Tr}(\tilde{C} H)+\operatorname{Tr}\left(L_{\tau}\left[\tau^{\prime}\right] \tilde{\tau}\right)\right]^{2}}{\operatorname{Tr}\left(L_{\tau}[\tilde{\tau}] \tilde{\tau}\right)} .
\end{aligned}
$$

At this stage, it is not obvious why the maximization of $\mathfrak{F}_{\mathrm{SQL}}\left(\mathcal{D}_{L, \omega}\right)$ over $C$ and $\tilde{C}$ is equal to $\mathfrak{F}_{\mathrm{SQL}}^{(u)}\left(\mathcal{E}_{\omega}\right)$. To see this, 
we need to reformulate the SQL upper bound using its dual program. First we note that

$$
\mathfrak{F}_{\mathrm{SQL}}^{(u)}\left(\mathcal{E}_{\omega}\right)=\max _{C: \operatorname{Tr}\left(C^{\dagger} C\right)=1} \min _{h: \beta=0} 4 \operatorname{Tr}\left(C^{\dagger} \alpha C\right),
$$

where we are allowed to exchange the orders of maximization and minimization thanks to Sion's minimax theorem $[96,97]$. Fixing $C$, we consider the optimization problem $\min _{h: \beta=0} 4 \operatorname{Tr}\left(C^{\dagger} \alpha C\right)$. When $C$ is full rank, we can show that it is equivalent to $\max _{\tilde{C} \in \mathbb{H}_{d}} f(C, \tilde{C})$, where $\tilde{C}$ is introduced as the Lagrange multiplier associated with the constraint $\beta=0$ [98] and the optimal $\tilde{C}$ is traceless.

The procedure to find a near-optimal code such that $\mathfrak{F}_{\mathrm{SQL}}\left(\mathcal{D}_{L, \omega}\right)>\mathfrak{F}_{\mathrm{SQL}}^{(u)}\left(\mathcal{E}_{\omega}\right)-\eta$ for any $\eta>0$ is as follows.

(1) Find a full rank $C^{\diamond}$ such that $\operatorname{Tr}\left(C^{\diamond \dagger} C^{\diamond}\right)=1$ and $\min _{h: \beta=0} 4 \operatorname{Tr}\left(C^{\diamond \dagger} \alpha C^{\diamond}\right)>\mathfrak{F}_{\mathrm{SQL}}^{(u)}\left(\mathcal{E}_{\omega}\right)-\eta / 2$.

(2) Find a Hermitian $\tilde{C}^{\diamond}$ such that $f\left(C^{\diamond}, \tilde{C}\right)$ is maximized and let $D^{\diamond}=\frac{1}{2} C^{\diamond-1} \tilde{C}^{\diamond}$. Rescale $D^{\diamond}$ such that $\operatorname{Tr}\left(D^{\diamond \dagger} D^{\diamond}\right)=1$.

(3) Calculate $\left.\quad \mathfrak{F}_{\mathrm{SQL}}\left(\mathcal{D}_{L, \omega}\right)\right|_{C=C^{\diamond}, D=D^{\diamond}} \quad$ using Eqs. (35)-(38) and Eq. (40). Find a small $\varepsilon^{\diamond}>0$ such that $\mathfrak{F}_{\mathrm{SQL}}\left(\mathcal{D}_{L, \omega}\right)>f\left(C^{\diamond}, \tilde{C}^{\diamond}\right)-\eta / 2$.

The numerical algorithms for steps (1) and (2) are provided in Appendix F, where the most computationally intensive part is a SDP. Note that in contrast to the HL case, here we require $2 d$-dimensional ancillae, twice as large as probes. In principle, however, $d$-dimensional ancillae are sufficient to achieve the asymptotic QFI, considering the Schmidt decomposition on the input state, though we no longer have explicit encoding and decoding protocols when using $d$-dimensional ancillae.

To conclude, we propose a perturbation code that can achieve the SQL upper bound with an arbitrarily small error. We take the limit where the parameter $\varepsilon$ that distinguishes the logical zero and one states is sufficiently small. Note that if we take $\varepsilon=0$, the probe state will be a product state and we can only achieve $\mathfrak{F}_{1}\left(\mathcal{D}_{L, \omega}\right)$. This discontinuity appears because we must first take the limit $N \rightarrow \infty$ before taking the limit $\varepsilon \rightarrow 0$ and the impact of a small $\varepsilon$ becomes significant in the asymptotic limit.

\section{EXAMPLES}

In this section, we provide three applications of our theorems. We first compute the asymptotic QFI of singlequbit depolarizing channels, which were not fully explored before. It is a case where $\mathfrak{F}_{\mathrm{SQL}}>\mathfrak{F}_{1}$ whenever the HNKS condition is violated. Second, we consider amplitude damping channels and obtain an analytical solution of a near-optimal QEC protocol. We directly see how the gap between the attainable QFI and $\mathfrak{F}_{\mathrm{SQL}}$ shrinks when $\varepsilon$ approaches 0 . In the third example, we consider a special type of channel that always satisfies $\mathfrak{F}_{\mathrm{SQL}}=\mathfrak{F}_{1}$ and provide a new simple proof of it.

\section{A. Single-qubit depolarizing channels}

Here we calculate $\mathfrak{F}_{1}, \mathfrak{F}_{\mathrm{SQL}}$, and $\mathfrak{F}_{\mathrm{HL}}$ for depolarizing channels $\mathcal{N}_{\omega}^{d}(\rho)=\mathcal{N}^{d}\left[\mathcal{U}_{\omega}(\rho)\right]$, where

$$
\mathcal{N}^{d}(\rho)=(1-p) \rho+p_{x} \sigma_{x} \rho \sigma_{x}+p_{y} \sigma_{y} \rho \sigma_{y}+p_{z} \sigma_{z} \rho \sigma_{z}
$$

$p_{x, y, z} \geq 0, p=p_{x}+p_{y}+p_{z}<1$, and $\mathcal{U}_{\omega}(\cdot)=e^{-i \omega \sigma / 2 z}(\cdot)$ $e^{i \omega \sigma_{z} / 2}$. The $p_{x, y, z}$ are independent of $\omega$.

First, we note that the HNKS condition is satisfied if and only if $p_{x}=p_{z}=0$ or $p_{y}=p_{z}=0$. When the HNKS condition is satisfied, $\mathfrak{F}_{\mathrm{HL}}\left(\mathcal{N}_{\omega}^{d}\right)=1$. It is the same as the $\mathfrak{F}_{\mathrm{HL}}$ when there is no noise $(p=0)$ because the Kraus operator $\left(\sigma_{x}\right.$ or $\left.\sigma_{y}\right)$ is perpendicular to the Hamiltonian $\left(\sigma_{z}\right)$ and could be fully corrected. It is consistent with previous results for single-qubit Hamiltonian estimation that the HL is achievable if and only if the Markovian noise is rank one and not parallel to the Hamiltonian [32,63-68]. As calculated in Appendix H,

$$
\mathfrak{F}_{1}\left(\mathcal{N}_{\omega}^{d}\right)=1-w
$$

where $w=4\left[p_{x} p_{y} /\left(p_{x}+p_{y}\right)+(1-p) p_{z} /\left(1-p+p_{z}\right)\right]$ $\leq 1$. When the HNKS condition is violated,

$$
\mathfrak{F}_{\mathrm{SQL}}\left(\mathcal{N}_{\omega}^{d}\right)=(1-w) / w
$$

In the equations above, when $p_{x}=p_{y}=0$, we take $p_{x} p_{y} /\left(p_{x}+p_{y}\right)=0$, in which case $\mathcal{N}_{\omega}^{d}$ becomes the dephasing channel introduced in Sec. III where $\phi=\omega$ and $p$ is independent of $\omega$.

We observe that

$$
\mathfrak{F}_{\mathrm{SQL}}\left(\mathcal{N}_{\omega}^{d}\right)=\mathfrak{F}_{1}\left(\mathcal{N}_{\omega}^{d}\right) / w \geq \mathfrak{F}_{1}\left(\mathcal{N}_{\omega}^{d}\right)
$$

and the equality $(w=1)$ holds if and only if $p_{x}=p_{y}$ and $p_{z}+p_{x}=\frac{1}{2}$, in which case $\mathfrak{F}_{\mathrm{SQL}}\left(\mathcal{N}_{\omega}^{d}\right)=\mathfrak{F}_{1}\left(\mathcal{N}_{\omega}^{d}\right)=0$, and $\mathcal{N}_{\omega}^{d}=\mathcal{N}^{d}$ becomes a mixture of a completely dephasing channel and a completely depolarizing channel [99] where $\omega$ cannot be detected.

In general, $\mathfrak{F}_{\mathrm{SQL}}\left(\mathcal{N}_{\omega}^{d}\right)$ is nonadditive. In particular, when $p \ll 1$, we have $w \ll 1$ and $\mathfrak{F}_{\mathrm{SQL}}\left(\mathcal{N}_{\omega}^{d}\right) \gg$ $\mathfrak{F}_{1}\left(\mathcal{N}_{\omega}^{d}\right)$. We also illustrate the difference between $\mathfrak{F}_{\mathrm{SQL}}\left(\mathcal{N}_{\omega}^{d}\right)$ and $\mathfrak{F}_{1}\left(\mathcal{N}_{\omega}^{d}\right)$ in Fig. 3 by plotting $\mathfrak{F}_{\mathrm{SQL}}\left(\mathcal{N}_{\omega}^{d}\right)$, $\mathfrak{F}_{1}\left(\mathcal{N}_{\omega}^{d}\right)$ as functions of $p_{x}$ and $p_{y}$ when $p_{z}=0.1$. At $\left(p_{x}, p_{y}, p_{z}\right)=(0.4,0.4,0.1), \mathfrak{F}_{\mathrm{SQL}}\left(\mathcal{N}_{\omega}^{d}\right)=\mathfrak{F}_{1}\left(\mathcal{N}_{\omega}^{d}\right)=0$. The ratio $\mathfrak{F}_{\mathrm{SQL}}\left(\mathcal{N}_{\omega}^{d}\right) / \mathfrak{F}_{1}\left(\mathcal{N}_{\omega}^{d}\right)$ increases near the boundary of $p_{x}+p_{y}<0.9$.

We remark here that, when the dimension of the system is large, for example, a qudit depolarizing channel or 


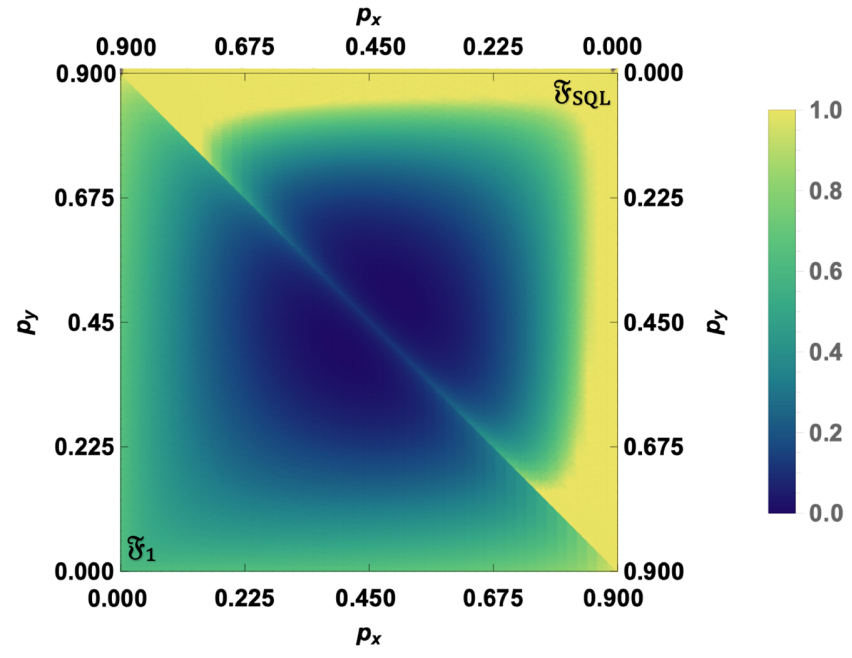

FIG. 3. Plots of $\mathfrak{F}_{1}\left(\mathcal{N}_{\omega}^{d}\right)$ and $\mathfrak{F}_{\mathrm{SQL}}\left(\mathcal{N}_{\omega}^{d}\right)$ as functions of $p_{x}$ and $p_{y}$ when $p_{z}=0.1$. The lower-left and upper-right parts are plots of $\mathfrak{F}_{1}\left(\mathcal{N}_{\omega}^{d}\right)$ and $\mathfrak{F}_{\mathrm{SQL}}\left(\mathcal{N}_{\omega}^{d}\right)$, respectively.

a collective dephasing channel [100], although $\mathfrak{F}_{1}, \mathfrak{F}_{\mathrm{SQL}}$ and the optimal input states can be found numerically via SDPs, analytical solutions may not exist. In that case, it might be helpful to compute analytical upper bounds on the QFI (see Appendix F of Ref. [101]) or consider the limit of large ensembles and use variational methods to solve for the QFI [30].

\section{B. Amplitude damping channels}

In the first example, we focus on computing the asymptotic QFI for single-qubit depolarizing channels, but we do not provide explicit QEC protocols achieving the QFI. Here we present a second example, where we obtain an analytical solution of the optimal QEC protocol and also analyze its performance when $\varepsilon$ is not a small constant.

Here we consider amplitude damping channels $\mathcal{N}_{\omega}^{\mathrm{AD}}(\rho)$ $=\mathcal{N}^{\mathrm{AD}}\left[\mathcal{U}_{\omega}(\rho)\right]$ defined by

$$
\mathcal{N}^{\mathrm{AD}}(\rho)=K_{1}^{\mathrm{AD}} \rho K_{1}^{\mathrm{AD} \dagger}+K_{2}^{\mathrm{AD}} \rho K_{2}^{\mathrm{AD} \dagger},
$$

where $K_{1}^{\mathrm{AD}}=|0\rangle\langle 0|+\sqrt{1-p}| 1\rangle\langle 1|$ and $K_{2}^{\mathrm{AD}}=\sqrt{p}|0\rangle$ $\langle 1|$, and $p$ represents the probability of a particle switching from $|1\rangle$ to $|0\rangle$ that is independent of $\omega$. Again, $\mathcal{U}_{\omega}$ is the Pauli- $Z$ rotation $e^{-i(\omega / 2) \sigma_{z}}$. We assume that $\omega=0$ in this section for simplicity because, for nonzero $\omega$, the QFI is the same and we only need to rotate the code accordingly.

As before, amplitude damping channels follow the SQL as long as $p>0$. Thus, we only focus on the situation where the HNKS condition is violated. As shown in Appendix I, $\mathfrak{F}_{\mathrm{SQL}}\left(\mathcal{N}_{\omega}^{\mathrm{AD}}\right)=4(1-p) / p[28]$ and the nearoptimal QEC protocol can be obtained using our algorithm from Sec. VI. The QEC code is characterized by two small but nonzero constants $\delta$ and $\varepsilon$, where $\delta$ is to make sure $C^{\diamond}$ is full rank [see step (1) of our algorithm in Sec. VI] and $\varepsilon=o(\delta)$ is the small constant in the perturbation code:

$$
\begin{aligned}
& \left|0_{L}\right\rangle=\sin (\delta+\varepsilon)|0\rangle_{\mathcal{P}}|00\rangle_{\mathcal{A}}+\cos (\delta+\varepsilon)|1\rangle_{\mathcal{P}}|10\rangle_{\mathcal{A}}, \\
& \left|1_{L}\right\rangle=\sin (\delta-\varepsilon)|0\rangle_{\mathcal{P}}|01\rangle_{\mathcal{A}}+\cos (\delta-\varepsilon)|1\rangle_{\mathcal{P}}|11\rangle_{\mathcal{A}} .
\end{aligned}
$$

Note that we use trigonometric functions instead of $\varepsilon$ and $\sqrt{1-\varepsilon^{2}}$ as before just to simplify the notation. We also need the optimal recovery channel, which is determined by

$$
G_{\mathrm{opt}}=\frac{2 i}{\sqrt{1-p}}|00\rangle\left\langle 11\left|+\frac{-2 i}{\sqrt{1-p}}\right| 11\right\rangle\langle 00| .
$$

The asymptotic channel QFI $\mathfrak{F}_{\mathrm{SQL}}\left(\mathcal{D}_{L, \omega}\right)$ attainable using the QEC protocol above is $\mathfrak{F}_{\mathrm{SQL}}\left(\mathcal{D}_{L, \omega}\right)=|\dot{\xi}|^{2} /\left(1-|\xi|^{2}\right)$, where

$$
\begin{gathered}
\xi=1-\frac{2 p \sin ^{2}(\delta)}{1-p} \varepsilon^{2}+O\left(\varepsilon^{4}\right), \\
\dot{\xi}=-2 i \sin (2 \delta) \varepsilon+O\left(\varepsilon^{3}\right),
\end{gathered}
$$

and

$$
\mathfrak{F}_{\mathrm{SQL}}\left(\mathcal{D}_{L, \omega}\right)=\frac{4(1-p) \cos ^{2}(\delta)}{p}+O\left(\varepsilon^{2}\right),
$$

which approaches $\mathfrak{F}_{\mathrm{SQL}}\left(\mathcal{N}_{\omega}^{\mathrm{AD}}\right)$ for small $\delta$. Note, however, that we cannot take $\delta=0$ because then $\dot{\xi}=0$. This means that $\mathfrak{F}_{\mathrm{SQL}}\left(\mathcal{N}_{\omega}^{\mathrm{AD}}\right)$ is achievable with an arbitrarily small but nonzero error. The exact values of $\xi$ and $\dot{\xi}$ as functions of $\delta$ and $\varepsilon$ can be found in Appendix I.

To visualize the gap between $\mathfrak{F}_{\mathrm{SQL}}\left(\mathcal{D}_{L, \omega}\right)$ and $\mathfrak{F}_{\mathrm{SQL}}$ $\left(\mathcal{N}_{\omega}^{\mathrm{AD}}\right)$, we plot it in Fig. 4 . We take $p=0.5,0.001$ in Figs. 4(a) and 4(b), and $\varepsilon=0.9 \delta, 0.5 \delta, 0.1 \delta, \varepsilon \rightarrow 0$ in each figure, and plot the ratio between the attainable QFI $\mathfrak{F}_{\mathrm{SQL}}\left(\mathcal{D}_{L, \omega}\right)$ and the optimal QFI $\mathfrak{F}_{\mathrm{SQL}}\left(\mathcal{N}_{\omega}^{\mathrm{AD}}\right)$ as a function of $\delta$. Figures 4(a) and 4(b) are almost identical, showing the robustness of our code against the change in noise rates. We also see that the curve from $\varepsilon=0.1 \delta$ almost overlaps with the limiting one [Eq. (59)] as $\varepsilon \rightarrow 0$. Moreover, we compare our ancilla-assisted QEC protocol with ancillafree protocols that achieve at most $\mathfrak{F}_{\mathrm{SQL}}\left(\mathcal{N}_{\omega}^{\mathrm{AD}}\right) / 4[30,31]$. It outperforms the optimal ancilla-free protocols in a large range (of $\delta$ and $\varepsilon$ ), showing the power of noiseless ancillae in phase estimation under amplitude damping noise. This type of phenomenon does not occur in dephasing channels where ancilla-free protocols are optimal $[30,31,39]$. 


$$
\frac{\mathfrak{F}_{\mathrm{SQL}}\left(\mathcal{D}_{\mathrm{L}, \omega}\right)}{\mathfrak{F}_{\mathrm{SQL}}\left(\mathcal{N}_{\omega}^{\mathrm{AD}}\right)} \quad \quad p=0.5
$$

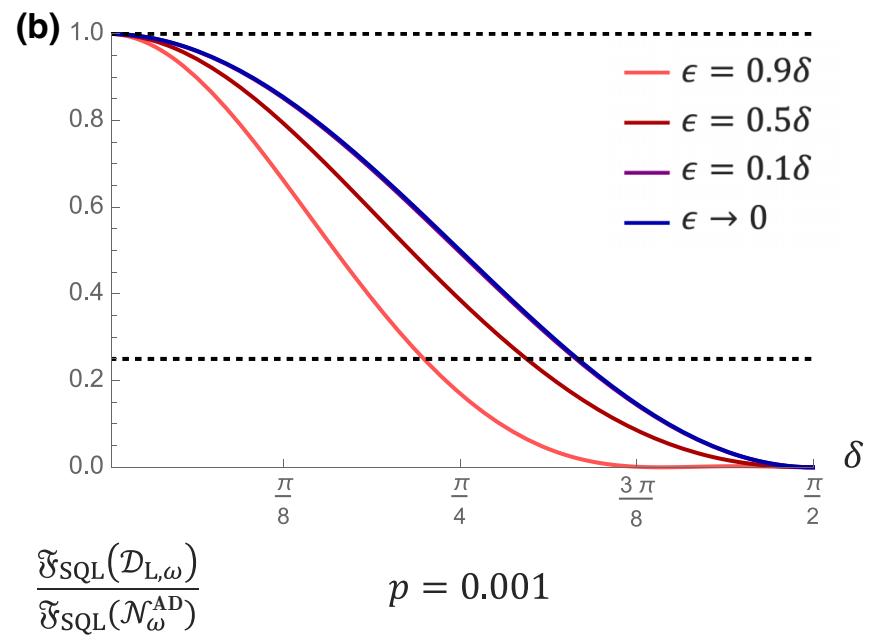

(a) 1

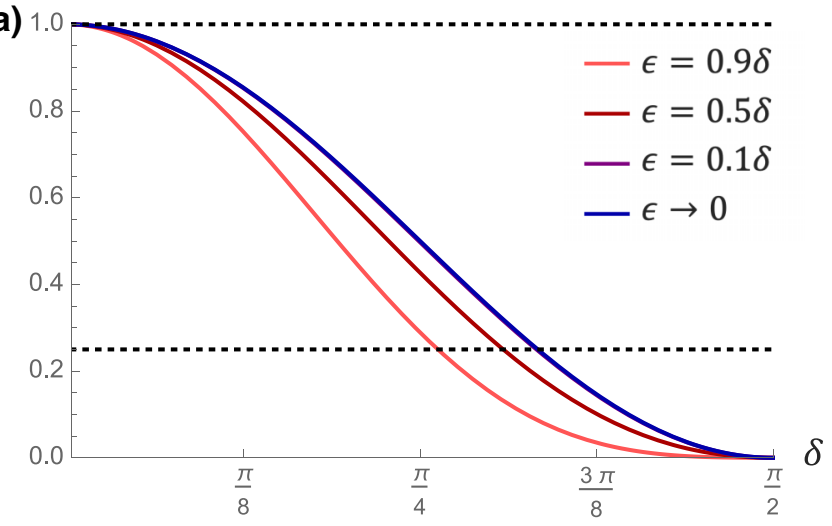

FIG. 4. Plots of $\mathfrak{F}_{\mathrm{SQL}}\left(\mathcal{D}_{L, \omega}\right) / \mathfrak{F}_{\mathrm{SQL}}\left(\mathcal{N}_{\omega}^{\mathrm{AD}}\right)$ as a function of $\delta$. We take $p=0.5,0.001$ in (a),(b) and $\varepsilon=0.9 \delta, 0.5 \delta, 0.1 \delta, \varepsilon \rightarrow 0$ in each figure. The curves from $\varepsilon=0.1 \delta$ and $\varepsilon \rightarrow 0$ are almost indistinguishable from each other. The dashed lines are at 1 and $\frac{1}{4}$, where the former represents the upper bound $\mathfrak{F}_{\mathrm{SQL}}\left(\mathcal{N}_{\omega}^{\mathrm{AD}}\right)$ and the latter represents the optimal asymptotic QFI without the assistance of ancillae $\mathfrak{F}_{\mathrm{SQL}}\left(\mathcal{N}_{\omega}^{\mathrm{AD}}\right) / 4$ [30,31]. Our QEC protocol outperforms the ancilla-free protocols even for large $\delta$ and $\varepsilon$.

\section{U-covariant channels}

Finally, we consider a special class of channel that satisfies $\mathfrak{F}_{\mathrm{SQL}}=\mathfrak{F}_{1}$. It covers many typical noise channels such as Pauli channels where noise rates are unknown, whereas in the first two examples we focus on phase estimation. Let $\mathbb{U}=\left\{U_{i}\right\}_{i=1}^{n} \subset \mathbb{C}^{d \times d}$ be a set of unitary operators such that, for some probability distribution $\left\{p_{i}\right\}_{i=1}^{n},\left\{\left(p_{i}, U_{i}\right)\right\}_{i=1}^{n}$ is a unitary 1-design [102], satisfying

$$
\sum_{i=1}^{n} p_{i} U_{i} A U_{i}^{\dagger}=\operatorname{Tr}(A) \frac{I}{d} \quad \text { for all } A \in \mathbb{C}^{d \times d}
$$

For example, when $\mathbb{U}$ is a unitary orthonormal basis of $\mathbb{C}^{d \times d},\left\{\left(1 / d^{2}, U_{i}\right)\right\}_{i=1}^{d^{2}}$ is a unitary 1-design. Given a quantum channel $\mathcal{T}_{\omega}(\cdot)=\sum_{i=1}^{r} K_{i}(\cdot) K_{i}^{\dagger}$, we call it $\mathbb{U}$-covariant if, for all $U \in \mathbb{U}$, there is a unitary $V$ such that

$$
\mathcal{T}_{\omega}\left(U \rho U^{\dagger}\right)=V \mathcal{T}_{\omega}(\rho) V^{\dagger}
$$

Note that here it is important that $U$ and $V$ are independent of $\omega$, a feature called joint covariance [58]. It can be shown that $\mathfrak{F}_{1}\left(\mathcal{T}_{\omega}\right)=\mathfrak{F}_{\mathrm{SQL}}\left(\mathcal{T}_{\omega}\right)$ when $\mathcal{T}_{\omega}$ is $\mathbb{U}$-covariant, using the teleportation simulation technique $[55,56,103,104]$. Here we provide an alternative proof using only the definitions of $\mathfrak{F}_{1}$ and $\mathfrak{F}_{\mathrm{SQL}}$ in the minimax formulation.

Let $h$ be a solution of $\min _{h} \max _{\rho} 4 \operatorname{Tr}(\rho \alpha)$. As explained in Appendix F, for every $\rho$ that is a solution of $\max _{\rho} \min _{h} 4 \operatorname{Tr}(\rho \alpha),(h, \rho)$ is a saddle point, i.e.,

$$
4 \operatorname{Tr}\left(\rho \alpha^{\star}\right) \leq 4 \operatorname{Tr}\left(\rho \alpha^{\star}\right) \leq 4 \operatorname{Tr}(\rho \star \alpha)
$$

for all $\rho$ and $h$, where $\alpha=\left.\alpha\right|_{h=h} \bullet$. Then $\left.\left|C^{\bullet}\right\rangle\right\rangle \in \mathcal{H}_{\mathcal{P}} \otimes$ $\mathcal{H}_{\mathcal{A}}$ is an optimal input state of a single quantum channel $\mathcal{T}_{\omega}$ if and only if $\rho^{\star}=C^{\star} C^{\dagger}$ satisfies Eq. (62). According to Eq. (61), if $\left.\left|C^{\star}\right\rangle\right\rangle$ is an optimal input, $\left.\left|U C^{\star}\right\rangle\right\rangle=$ $(U \otimes I)\left|C^{\diamond}\right\rangle$ is also an optimal input for all $U \in \mathbb{U}$ and satisfies Eq. (62). Then $\sum_{i=1}^{n} p_{i} U_{i} \rho^{\bullet} U_{i}^{\dagger}=I / d$ also satisfies Eq. (62), implying that the maximally entangled state $|I / d\rangle\rangle$ is an optimal input for $\mathcal{T}_{\omega}$. The discussion above also works for $\mathcal{T}_{\omega}^{\otimes N}$ because $\mathcal{T}_{\omega}^{\otimes N}$ is $\mathbb{U}^{\otimes N}$-covariant and $\left\{\left(\Pi_{k} p_{i_{k}}, \otimes_{k} U_{i_{k}}\right)\right\}$ is a unitary 1-design on $\mathbb{C}^{N d \times N d}$. Therefore, $\left.\left|I / d^{N}\right\rangle\right\rangle$ is an optimal input for $\mathcal{T}_{\omega}^{\otimes N}$, which implies that $\mathfrak{F}_{N}\left(\mathcal{T}_{\omega}\right)=N \mathfrak{F}_{1}\left(\mathcal{T}_{\omega}\right)$.

\section{CONCLUSIONS AND OUTLOOK}

In this paper, we focus on the asymptotic behavior of the QFI of a quantum channel when the number of identical channels $N$ is infinitely large. We consolidate the HNKS condition by showing it unambiguously determines whether or not the scaling of the asymptotic QFI is quadratic or linear. In both cases, we show that the optimal input state achieving the asymptotic QFI could be found via a SDP. To find the optimal input state, we reduce every quantum channel to a single-qubit dephasing channel where both the phase and the noise parameter vary with respect to the unknown parameter and then optimize the asymptotic QFI of the logical dephasing channel over the encoding and the recovery channel. The optimal input state is either the logical GHZ state (when the HNKS condition is satisfied) or the logical spin-squeezed state (when the HNKS condition is violated). This provides a unified framework for channel estimation while previous results were centered on either Hamiltonian estimation or noise estimation in special situations.

Furthermore, our results imply that, when the HNKS condition is violated, sequential strategies provide no 
advantage over parallel strategies asymptotically, answering another open problem in quantum metrology. The regularized channel QFI $\mathfrak{F}_{\mathrm{SQL}}\left(\mathcal{E}_{\omega}\right)$ is a useful informationtheoretic measure and was recently shown to be useful in deriving bounds in covariant QEC [101,105]. It can also serve as a useful benchmark for practical quantum metrological tasks - one could compare the attainable Fisher information with $\mathfrak{F}_{\mathrm{SQL}}\left(\mathcal{E}_{\omega}\right)$ to determine how far a metrological protocol is from optimal. Moreover, we propose a two-dimensional QEC protocol to achieve $\mathfrak{F}_{\mathrm{SQL}}\left(\mathcal{E}_{\omega}\right)$, where the optimal input state is a concatenation of many-body spin-squeezed states and two-dimensional QEC codes (Fig. 2). This allows us to reduce the optimization in the entire Hilbert space that is exponentially large to that in a local Hilbert space, providing a new inspiration for numerical methods in quantum metrology [51,72,106-108].

It is left open whether sequential strategies provide no advantage over parallel strategies asymptotically when the HNKS condition is satisfied. The statement was proven true only for unitary channels [23], but there is still a gap between $\mathfrak{F}_{\mathrm{HL}}\left(\mathcal{E}_{\omega}\right)$ and state-of-the-art upper bounds on $\mathfrak{F}_{\mathrm{HL}}^{(\text {seq) }}\left(\mathcal{E}_{\omega}\right)$ for general quantum channels $[31,32,35,36]$. Note that, for multiparameter estimation, a gap between parallel strategies and sequential strategies exists even for unitary channels [109].

\section{ACKNOWLEDGMENTS}

We thank Chang-Ling Zou, Mark Wilde, Haining Pan, Zi-Wen Liu, Yuxiang Yang, Rafal DemkowiczDobrzanśki, Vishal Katariya, and Yu Chen for helpful discussions. We acknowledge support from the ARLCDQI (Grant No. W911NF-15-2-0067), ARO (Grants No. W911NF-18-1-0020 and No. W911NF-18-1-0212), ARO MURI (Grant No. W911NF-16-1-0349), AFOSR MURI (Grants No. FA9550-15-1-0015 and No. FA9550-19-10399), DOE (Grant No. DE-SC0019406), NSF (Grants No. EFMA-1640959 and No. OMA-1936118), and the Packard Foundation (Grant No. 2013-39273).

\section{APPENDIX A: DERIVING THE UPPER BOUND ON $\mathfrak{F}_{N}\left(\mathcal{E}_{\omega}\right)$}

For completeness, we provide a proof [24] of Eq. (8). Let $K_{i}^{(1)}=K_{i}$ for $i \in[r]$, where $[r]=\{1,2, \ldots, r\}$. Inductively, let

$$
K_{\iota}^{(n+1)}=K_{\iota_{1}}^{(n)} \otimes K_{\iota_{2}}^{(1)} \quad \text { for all } \iota=\left(\iota_{1}, \iota_{2}\right) \in[r]^{n} \times[r],
$$

where $\left\{K_{\iota}^{(n)}\right\}_{\iota \in[r]^{n}}$ is a Kraus representation of $\mathcal{E}_{\omega}^{\otimes n}$ for all $n$. Then let $\alpha^{(n)}=\sum_{\iota_{1}} \dot{K}_{\iota_{1}}^{(n) \dagger} \dot{K}_{\iota_{1}}^{(n)}, \beta^{(n)}=i \sum_{\iota_{1}} K_{\iota_{1}}^{(n) \dagger} \dot{K}_{\iota_{1}}^{(n)}$. We have

$$
\begin{aligned}
\alpha^{(n+1)} & =\sum_{\iota_{1}, l_{2}}\left(\frac{\partial\left(K_{\iota_{1}}^{(n)} \otimes K_{\iota_{2}}^{(1)}\right)}{\partial \omega}\right)^{\dagger}\left(\frac{\partial\left(K_{\iota_{1}}^{(n)} \otimes K_{\iota_{2}}^{(1)}\right)}{\partial \omega}\right) \\
& =\alpha^{(n)} \otimes I+2 \beta^{(n)} \otimes \beta^{(1)}+I \otimes \alpha^{(1)}, \\
\beta^{(n+1)} & =i \sum_{\iota_{1}, l_{2}}\left(\frac{\partial\left(K_{\iota_{1}}^{(n)} \otimes K_{\iota_{2}}^{(1)}\right)}{\partial \omega}\right)^{\dagger}\left(K_{\iota_{1}}^{(n)} \otimes K_{\iota_{2}}^{(1)}\right) \\
& =\beta^{(n)} \otimes I+I \otimes \beta^{(1)} .
\end{aligned}
$$

The solution is $\beta^{(N)}=\sum_{k=0}^{N-1} I^{\otimes k} \otimes \beta^{(1)} \otimes I^{\otimes N-1-k}$ and

$$
\begin{aligned}
\alpha^{(N)}= & \sum_{k=0}^{N-1} I^{\otimes k} \otimes \alpha^{(1)} \otimes I^{\otimes N-1-k}+2 \sum_{k_{1}=0}^{N-2} \sum_{k_{2}=0}^{N-2-k_{1}} I^{\otimes k_{1}} \\
& \otimes \beta^{(1)} \otimes I^{\otimes k_{2}} \otimes \beta^{(1)} \otimes I^{\otimes N-2-k_{1}-k_{2}} .
\end{aligned}
$$

Therefore, $\mathfrak{F}_{N}\left(\mathcal{E}_{\omega}\right) \leq 4\left\|\alpha^{(N)}\right\| \leq 4 N\left\|\alpha^{(1)}\right\|+4 N(N-1)$ $\left\|\beta^{(1)}\right\|^{2}$ and the inequality holds for any Kraus representation of $\mathcal{E}_{\omega}$. We can choose $\mathbf{K}^{\prime}=u \mathbf{K}$, then

$$
\mathfrak{F}_{N}\left(\mathcal{E}_{\omega}\right) \leq 4 \min _{h}\left[N\|\alpha\|+N(N-1)\|\beta\|^{2}\right],
$$

where $h=i u^{\dagger} \dot{u}$ is an arbitrary Hermitian matrix, $\alpha=$ $\dot{\mathbf{K}}^{\prime \dagger} \dot{\mathbf{K}}^{\prime}=(\dot{\mathbf{K}}-i h \mathbf{K})^{\dagger}(\dot{\mathbf{K}}-i h \mathbf{K})$, and $\beta=i \mathbf{K}^{\prime \dagger} \dot{\mathbf{K}}^{\prime}=i \mathbf{K}^{\dagger}$ $(\dot{\mathbf{K}}-i h \mathbf{K})$.

\section{APPENDIX B: CALCULATING THE QFI UPPER BOUNDS FOR DEPHASING CHANNELS}

Here we calculate $\mathfrak{F}_{\mathrm{HL}}^{(u)}=4 \min _{h}\|\beta\|^{2}$ and $\mathfrak{F}_{\mathrm{SQL}}^{(u)}=$ $4 \min _{h: \beta=0}\|\alpha\|$ for dephasing channels

$$
\begin{aligned}
\mathcal{D}_{\omega}(\rho) & =(1-p) e^{-i \phi \sigma_{z} / 2} \rho e^{i \phi \sigma_{z} / 2}+p \sigma_{z} e^{-i \phi \sigma_{z} / 2} \rho e^{i \phi \sigma_{z} / 2} \sigma_{z} \\
& =\sum_{i=1}^{2} K_{i} \rho K_{i}^{\dagger},
\end{aligned}
$$

where $K_{1}=\sqrt{1-p} e^{-i \phi \sigma_{z} / 2}, K_{2}=\sqrt{p} \sigma_{z} e^{-i \phi \sigma_{z} / 2}$. Assume that $p>0$; then

$$
\mathbf{K}=\left(\begin{array}{c}
\sqrt{1-p} e^{-i \phi \sigma_{z} / 2} \\
\sqrt{p} \sigma_{z} e^{-i \phi \sigma_{z} / 2}
\end{array}\right)
$$

$$
\dot{\mathbf{K}}=\left(\begin{array}{c}
\left(\frac{-\dot{p}}{2 \sqrt{1-p}}-\sqrt{1-p} \frac{i \dot{\phi}}{2} \sigma_{z}\right) e^{-i \phi \sigma_{z} / 2} \\
\left(\frac{\dot{p}}{2 \sqrt{p}}-\sqrt{p} \frac{i \dot{\phi}}{2} \sigma_{z}\right) e^{-i \phi \sigma_{z} / 2} \sigma_{z}
\end{array}\right),
$$




$$
\begin{gathered}
\dot{\mathbf{K}}-i h \mathbf{K}=\left(\begin{array}{c}
\left(\frac{-\dot{p}}{2 \sqrt{1-p}}-i h_{11} \sqrt{1-p}-\sqrt{1-p} \frac{i \dot{\phi}}{2} \sigma_{z}-i h_{12} \sqrt{p} \sigma_{z}\right) e^{-i \phi \sigma_{z} / 2} \\
\left(\frac{\dot{p}}{2 \sqrt{p}} \sigma_{z}-i h_{22} \sqrt{p} \sigma_{z}-\sqrt{p} \frac{i \dot{\phi}}{2}-i h_{21} \sqrt{1-p}\right) e^{-i \phi \sigma_{z} / 2}
\end{array}\right), \\
\beta=i \mathbf{K}^{\dagger}(\dot{\mathbf{K}}-i h \mathbf{K})=\frac{\dot{\phi}}{2} \sigma_{z}+(1-p) h_{11}+p h_{22}+\sqrt{p(1-p)}\left(h_{12}+h_{21}\right) \sigma_{z}, \\
\alpha=(\dot{\mathbf{K}}-i h \mathbf{K})^{\dagger}(\dot{\mathbf{K}}-i h \mathbf{K}) \\
=\frac{\dot{p}^{2}}{4 p(1-p)}+h_{11}^{2}(1-p)+h_{22}^{2} p+\frac{\dot{\phi}^{2}}{4}+\left|h_{12}\right|^{2}+2 \sqrt{p(1-p)} \dot{\phi} \operatorname{Re}\left[h_{12}\right] \\
+2 \operatorname{Re}\left[-\frac{\dot{p} \sqrt{p}}{\sqrt{1-p}} i h_{12}+\left[(1-p) h_{11}+h_{22} p\right] \frac{\dot{\phi}}{2}+\left(h_{11} h_{12}+h_{22} h_{21}\right) \sqrt{p(1-p)}-i \frac{\dot{p} \sqrt{1-p}}{\sqrt{p}} h_{21}\right] \sigma_{z} .
\end{gathered}
$$

Here $\beta=0$ is equivalent to $(1-p) h_{11}+p h_{22}=0$ and $\dot{\phi} / 2+\sqrt{p(1-p)}\left(h_{12}+h_{21}\right)=0$, which is achievable for any $p>0$. When $h_{11}=h_{22}=0$ and $h_{12}=h_{21}=$ $-\dot{\phi} / 4 \sqrt{p(1-p)}, \quad\|\alpha\|=\min _{h: \beta=0}\|\alpha\|=(1-2 p)^{2} \dot{\phi}^{2} /$ $[16 p(1-p)]+\dot{p}^{2} /[4(1-p) p]$. Then

$$
\begin{aligned}
\mathfrak{F}_{\mathrm{SQL}}^{(u)}\left(\mathcal{D}_{\omega}\right)=4 \min _{h: \beta=0}\|\alpha\| & =\frac{(1-2 p)^{2} \dot{\phi}^{2}}{4 p(1-p)}+\frac{\dot{p}^{2}}{(1-p) p} \\
& =\frac{|\dot{\xi}|^{2}}{1-|\xi|^{2}},
\end{aligned}
$$

where $\xi=(1-2 p) e^{-i \phi}=\left\langle 0\left|\mathcal{D}_{\omega}(|0\rangle\langle 1|)\right| 1\right\rangle$ is a complex number completely determining the channel.

When $p=0$, we must also have $\dot{p}=0$. Then $\beta=$ $\dot{\phi} \sigma_{z} / 2+h_{11}$ and

$$
\mathfrak{F}_{\mathrm{HL}}^{(u)}\left(\mathcal{D}_{\omega}\right)=4 \min _{h}\|\beta\|^{2}=|\dot{\phi}|^{2}=|\dot{\xi}|^{2}
$$

We can also calculate the channel QFI

$$
\begin{aligned}
\mathfrak{F}_{1}^{(u)}\left(\mathcal{D}_{\omega}\right) & =4 \min _{h}\|\alpha\| \\
& = \begin{cases}(1-2 p)^{2} \dot{\phi}^{2}+\frac{\dot{p}^{2}}{(1-p) p}, & p>0, \\
(1-2 p)^{2} \dot{\phi}^{2}, & p=0 .\end{cases}
\end{aligned}
$$

This can be achieved using $\left|\psi_{0}\right\rangle=(|0\rangle+|1\rangle) / \sqrt{2}$.

\section{APPENDIX C: A USEFUL FORMULA FOR CALCULATING THE QFI OF DEPHASING CHANNELS}

In this appendix, we prove Eq. (17). Let $|\psi\rangle=$ $e^{-i \phi J_{z}}\left|\psi_{0}\right\rangle$, and let a subspace

$$
\mathcal{Z}=\operatorname{span}\left\{\prod_{k=1}^{N}\left(\sigma_{z}^{(k)}\right)^{j_{k}}|\psi\rangle,\left(j_{1}, \ldots, j_{N}\right) \in\{0,1\}^{N}\right\} .
$$

Assume that $\operatorname{dim} \mathcal{Z}=n$. Here $\mathcal{Z}$ must have an orthonormal basis $\left\{\left|e_{\ell}\right\rangle\right\}_{\ell=1}^{n}$, where $\left|e_{\ell}\right\rangle=\sum_{j_{1}, \ldots, j_{N}=0}^{1} r_{\ell,\left(j_{1}, \ldots, j_{N}\right)}$ $\prod_{k=1}^{N}\left(\sigma_{z}^{(k)}\right)^{j_{k}}|\psi\rangle$ with real $r_{\ell,\left(j_{1}, \ldots, j_{N}\right)}$. For example, one can use the Gram-Schmidt procedure to find $\left\{\left|e_{\ell}\right\rangle\right\}_{\ell=1}^{n}$ because $\left\langle\psi\left|\prod_{k=1}^{N}\left(\sigma_{z}^{(k)}\right)^{j_{k}}\right| \psi\right\rangle \in \mathbb{R}$ for all $\left(j_{1}, \ldots, j_{N}\right) \in\{0,1\}^{\otimes N}$.

Then

$$
\begin{aligned}
\rho_{\omega}= & \mathcal{D}_{\omega}^{\otimes N}\left(\left|\psi_{0}\right\rangle\left\langle\psi_{0}\right|\right) \\
= & \left(\left.\mathcal{D}_{\omega}\right|_{\phi=0}\right)^{\otimes N}(|\psi\rangle\langle\psi|) \\
= & \sum_{j_{1}, \ldots, j_{N}=0}^{1}(1-p)^{\left(N-\sum_{k=1}^{N} j_{k}\right)} p^{\left(\sum_{k=1}^{N} j_{k}\right)} \\
& \times \prod_{k=1}^{N}\left(\sigma_{z}^{(k)}\right)^{j_{k}}|\psi\rangle\langle\psi| \prod_{k=1}^{N}\left(\sigma_{z}^{(k)}\right)^{j_{k}} \\
= & \sum_{\ell, \ell^{\prime}=1}^{n} \chi_{\ell \ell^{\prime}}\left|e_{\ell}\right\rangle\left\langle e_{\ell^{\prime}}\right|,
\end{aligned}
$$

where $\chi \in \mathbb{R}^{n \times n}$ is a symmetric matrix; $\chi=\sum_{i=1}^{n} \mu_{i} v_{i} v_{i}^{T}$, where the $v_{i}$ are real orthonormal eigenvectors of $\chi$. Then we can write $\rho_{\omega}=\sum_{\ell=1}^{n} \mu_{\ell}\left|\psi_{\ell}\right\rangle\left\langle\psi_{\ell}\right|$, where $\left|\psi_{\ell}\right\rangle=\sum_{\ell^{\prime}=1}^{n} v_{\ell \ell^{\prime}}\left|e_{\ell^{\prime}}\right\rangle$. Then, according to the definition of 
QFI,

$$
F\left(\rho_{\omega}\right)=2 \sum_{\ell \ell^{\prime}: \mu_{\ell}+\mu_{\ell^{\prime}} \neq 0} \frac{\left|\left\langle\psi_{\ell}\left|\dot{\rho}_{\omega}\right| \psi_{\ell^{\prime}}\right\rangle\right|^{2}}{\mu_{\ell}+\mu_{\ell^{\prime}}} .
$$

Note that, in principle, Eq. (C3) only holds when $\left\{\left|\psi_{\ell}\right\rangle\right\}$ is a complete basis of $\mathcal{H}_{\mathcal{P}}^{\otimes N}$, that is, $\operatorname{span}\left\{\left|\psi_{\ell}\right\rangle\right\}=$
$\mathcal{H}_{\mathcal{P}}^{\otimes N}$. However, we are allowed to restrict the summation on the right-hand side of Eq. (C3) to states in the subspace $\mathcal{Z}$, i.e., $\operatorname{span}\left\{\left|\psi_{\ell}\right\rangle\right\}=\mathcal{Z}$, because $\Pi_{\mathcal{Z}} \rho_{\omega} \Pi_{\mathcal{Z}}=\rho_{\omega}$ and $\Pi_{\mathcal{Z}} \dot{\rho}_{\omega} \Pi_{\mathcal{Z}}=\dot{\rho}_{\omega}$, i.e., any state perpendicular to $\mathcal{Z}$ does not contribute to the QFI.

The derivative of $\rho_{\omega}$ with respect to $\omega$ is

$$
\begin{aligned}
\dot{\rho}_{\omega}= & \frac{\partial \rho_{\omega}}{\partial p} \dot{p}+\frac{\partial \rho_{\omega}}{\partial \phi} \dot{\phi} \\
= & \sum_{j_{1}, \ldots, j_{N}=0}^{1} \frac{\partial(1-p)^{\left(N-\sum_{k=1}^{N} j_{k}\right)} p^{\left(\sum_{k=1}^{N} j_{k}\right)}}{\partial \omega} \prod_{k=1}^{N}\left(\sigma_{z}^{(k)}\right)^{j_{k}}|\psi\rangle\langle\psi| \prod_{k=1}^{N}\left(\sigma_{z}^{(k)}\right)^{j_{k}} \\
& +\sum_{j_{1}, \ldots, j_{N}=0}^{1}(1-p)^{\left(N-\sum_{k=1}^{N} j_{k}\right)} p^{\left(\sum_{k=1}^{N} j_{k}\right)} \prod_{k=1}^{N}\left(\sigma_{z}^{(k)}\right)^{j_{k}} \frac{\partial|\psi\rangle\langle\psi|}{\partial \omega} \prod_{k=1}^{N}\left(\sigma_{z}^{(k)}\right)^{j_{k}}
\end{aligned}
$$

Then we have

$$
\left\langle\psi_{\ell}\left|\dot{\rho}_{\omega}\right| \psi_{\ell^{\prime}}\right\rangle=a_{\ell \ell^{\prime}}+i b_{\ell \ell^{\prime}},
$$

where $\quad a_{\ell \ell^{\prime}}=\left\langle\psi_{\ell}\left|\left(\partial \rho_{\omega} / \partial p\right) \dot{p}\right| \psi_{\ell^{\prime}}\right\rangle \in \mathbb{R}, \quad b_{\ell \ell^{\prime}}=-i$ $\left\langle\psi_{\ell}\left|\left(\partial \rho_{\omega} / \partial \phi\right) \dot{\phi}\right| \phi_{\ell^{\prime}}\right\rangle \in \mathbb{R}$. Therefore,

$$
\begin{aligned}
F\left(\rho_{\omega}\right) & =2 \sum_{\ell \ell^{\prime}: \mu_{\ell}+\mu_{\ell^{\prime}} \neq 0} \frac{\mid\left\langle\left.\psi_{\ell}\left|\dot{\rho}_{\omega}\right| \psi_{\ell^{\prime}}\right|^{2}\right.}{\mu_{\ell}+\mu_{\ell^{\prime}}} \\
& =2 \sum_{\ell \ell^{\prime}: \mu_{\ell}+\mu_{\ell^{\prime}} \neq 0} \frac{\left|a_{\ell \ell^{\prime}}\right|^{2}+\left|b_{\ell \ell^{\prime}}\right|^{2}}{\mu_{\ell}+\mu_{\ell^{\prime}}}=F_{p}\left(\rho_{\omega}\right)+F_{\phi}\left(\rho_{\omega}\right),
\end{aligned}
$$

which is the same as Eq. (17).

\section{APPENDIX D: OPTIMAL SQUEEZED STATE FOR DEPHASING CHANNELS}

Let the input state $\left|\psi_{0}\right\rangle=e^{i \phi J_{z}}\left|\psi_{\mu, \nu}\right\rangle$, where $\left|\psi_{\mu, \nu}\right\rangle$ is an $N$-qubit spin-squeezed state

$$
\left|\psi_{\mu, \nu}\right\rangle=e^{-i \nu J_{x}} e^{-i \mu J_{z}^{2} / 2} e^{-i \pi J_{y} / 2}|0\rangle^{\otimes N} .
$$

The output state is $\rho_{\omega}=\mathcal{D}_{\omega}^{\otimes N}\left(\left|\psi_{0}\right\rangle\left\langle\psi_{0}\right|\right)=\left(\left.\mathcal{D}_{\omega}\right|_{\phi=0}\right)^{\otimes N}$ $(|\psi\rangle\langle\psi|)$. Then

$$
\begin{gathered}
\left\langle J_{x, y}\right\rangle_{\rho_{\omega}}=(1-2 p)\left\langle J_{x, y}\right\rangle_{\left|\psi_{\mu, \nu}\right\rangle}, \\
\left\langle J_{x, y}^{2}\right\rangle_{\rho_{\omega}}=\frac{N}{4}+(1-2 p)^{2}\left(\left\langle J_{x, y}^{2}\right\rangle_{\left|\psi_{\mu, \nu}\right\rangle}-\frac{N}{4}\right),
\end{gathered}
$$

$$
\frac{\partial\left\langle J_{x}\right\rangle_{\rho_{\omega}}}{\partial p} \dot{p}=-2 \dot{p}\left\langle J_{x}\right\rangle_{\left|\psi_{\mu, \nu}\right\rangle}
$$

$$
\frac{\partial\left\langle J_{y}\right\rangle_{\rho_{\omega}}}{\partial \phi} \dot{\phi}=(1-2 p) \dot{\phi}\left\langle J_{x}\right\rangle_{\left|\psi_{\mu, \nu}\right\rangle} .
$$

It was shown in Ref. [94] that, choosing $v=\pi / 2-$ $\frac{1}{2} \arctan (b / a)$,

$$
\left\langle J_{x}\right\rangle_{\left|\psi_{\mu, \nu}\right\rangle}=\frac{N}{2} \cos \left(\frac{\mu}{2}\right)^{N-1}, \quad\left\langle J_{y}\right\rangle_{\left|\psi_{\mu, \nu}\right\rangle}=0,
$$

$$
\begin{aligned}
& \left\langle\Delta J_{x}^{2}\right\rangle_{\left|\psi_{\mu, \nu}\right\rangle} \\
& =\frac{N}{4}\left[N\left(1-\cos ^{2(N-1)} \frac{\mu}{2}\right)-\left(\frac{N-1}{2}\right) a\right]
\end{aligned}
$$

$$
\left\langle\Delta J_{y}^{2}\right\rangle_{\left|\psi_{\mu, \nu}\right\rangle}=\frac{N}{4}\left[1+\frac{N-1}{4}\left(a-\sqrt{a^{2}+b^{2}}\right)\right]
$$

where $a=1-\cos ^{N-2} \mu, \quad b=4 \sin (\mu / 2) \cos ^{N-2}(\mu / 2)$. Let $N \gg 1, \mu=\Theta\left(N^{-5 / 6}\right)$. Then

$$
\begin{aligned}
\left\langle J_{x}\right\rangle_{\left|\psi_{\mu, \nu}\right\rangle} & \approx \frac{N}{2}, \quad\left\langle\Delta J_{x}^{2}\right\rangle_{\left|\psi_{\mu, \nu}\right\rangle} \approx O\left(N^{2 / 3}\right), \\
\left\langle\Delta J_{y}^{2}\right\rangle_{|\psi \mu, \nu\rangle} & \approx O\left(N^{2 / 3}\right),
\end{aligned}
$$


and $\left\langle\Delta J_{x}^{2}\right\rangle_{\rho_{\omega}} \approx\left\langle\Delta J_{y}\right\rangle_{\rho_{\omega}}^{2} \approx p(1-p) N,\left(\partial\left\langle J_{x}\right\rangle_{\rho_{\omega}} / \partial p\right) \dot{p} \approx$ $-\dot{p} N$, and $\left(\partial\left\langle J_{y}\right\rangle_{\rho_{\omega}} / \partial \phi\right) \dot{\phi} \approx(1-2 p) \dot{\phi} N / 2$.

\section{APPENDIX E: OPTIMIZING THE QFI WHEN THE HNKS CONDITION IS VIOLATED}

In this appendix, we optimize the QFI

$$
\mathfrak{F}_{\mathrm{SQL}}\left(\mathcal{D}_{L, \omega}\right)=\frac{|\dot{\xi}|^{2}}{1-|\xi|^{2}}
$$

using Eqs. (37)-(38). We expand $T$ and $E_{0} E_{1}^{\dagger}$ around $\varepsilon=$ 0 , i.e.,

$$
T=e^{i \varepsilon G}=1+i \varepsilon G-\frac{\varepsilon^{2}}{2} G^{2}+O\left(\varepsilon^{3}\right)
$$

$$
\begin{aligned}
E_{0} E_{1}^{\dagger} & =\left(1-\varepsilon^{2}\right) E E^{\dagger}-\varepsilon \sqrt{1-\varepsilon^{2}}\left(E F^{\dagger}-F E^{\dagger}\right)-\varepsilon^{2} F F^{\dagger} \\
& =\sigma-i \varepsilon \tilde{\sigma}-\varepsilon^{2}\left(F F^{\dagger}+E E^{\dagger}\right)+O\left(\varepsilon^{3}\right),
\end{aligned}
$$

where $\sigma=E E^{\dagger}$ and $\tilde{\sigma}=i\left(F E^{\dagger}-E F^{\dagger}\right)$. Then

$$
\begin{aligned}
\operatorname{Tr}\left(T E_{0} E_{1}^{\dagger}\right)= & 1-2 \varepsilon^{2}-\frac{\varepsilon^{2}}{2} \operatorname{Tr}\left(G^{2} \sigma\right) \\
& -i \varepsilon \operatorname{Tr}(G \sigma)-\varepsilon^{2} \operatorname{Tr}(G \tilde{\sigma})+O\left(\varepsilon^{3}\right)
\end{aligned}
$$

$$
\operatorname{Tr}\left[T\left(\dot{E}_{0} E_{1}^{\dagger}+E_{0} \dot{E}_{1}^{\dagger}\right)\right]=i \varepsilon \operatorname{Tr}(G \dot{\sigma})+O\left(\varepsilon^{2}\right)
$$

where we have used $\operatorname{Tr}\left(F^{\dagger} F\right)=1$ and $\operatorname{Tr}(\tilde{\sigma})=0$ because $\operatorname{Tr}\left(E^{\dagger} F\right)=0$. Then

$$
\begin{aligned}
\mathfrak{F}_{\mathrm{SQL}}\left(\mathcal{D}_{L, \omega}\right) & =\max _{G} \frac{|\operatorname{Tr}(G \dot{\sigma})|^{2}}{4-2 \operatorname{Tr}(G \tilde{\sigma})+\operatorname{Tr}\left(G^{2} \sigma\right)-|\operatorname{Tr}(G \sigma)|^{2}}+O(\varepsilon) \\
& =\max _{G, x} \frac{|\operatorname{Tr}(G \dot{\sigma})|^{2}}{4 x^{2}+2 x \operatorname{Tr}(G \tilde{\sigma})+\operatorname{Tr}\left(G^{2} \sigma\right)-|\operatorname{Tr}(G \sigma)|^{2}}+O(\varepsilon) \\
& =\max _{G} \frac{|\operatorname{Tr}(G \dot{\sigma})|^{2}}{-|\operatorname{Tr}(G \tilde{\sigma})|^{2} / 4+\operatorname{Tr}\left(G^{2} \sigma\right)-|\operatorname{Tr}(G \sigma)|^{2}}+O(\varepsilon),
\end{aligned}
$$

shown as Eq. (39) in the main text, where in the second line we have used the fact that any rescaling of $G(G \leftarrow-G / x)$ should not change the optimal QFI. Note that to obtain the solution of the original $G$ in $T$, we need to rescale the final solution back using $G \leftarrow 4 G / \operatorname{Tr}(G \tilde{\sigma})$.

To find the optimal $G$, we first observe that $\operatorname{Tr}(\dot{\sigma})=\operatorname{Tr}(\tilde{\sigma})=0$. Therefore, without loss of generality, we assume that $\operatorname{Tr}(G \sigma)=0$ because $G \leftarrow G-\operatorname{Tr}(G) I / r$ does not change the target function. Let the derivative of Eq. (E6) be zero. Then we have

$$
\begin{aligned}
& 2 \dot{\sigma}\left(\operatorname{Tr}\left(G^{2} \sigma\right)-\frac{|\operatorname{Tr}(G \tilde{\sigma})|^{2}}{4}\right)-\operatorname{Tr}(G \dot{\sigma})\left[(\sigma G+G \sigma)-\frac{2 \operatorname{Tr}(G \tilde{\sigma}) \tilde{\sigma}}{4}\right]=0 \\
\Longleftrightarrow & \frac{\dot{\sigma}}{\operatorname{Tr}(G \dot{\sigma})}\left(\operatorname{Tr}\left(G^{2} \sigma\right)-\frac{|\operatorname{Tr}(G \tilde{\sigma})|^{2}}{4}\right)+\frac{\operatorname{Tr}(G \tilde{\sigma}) \tilde{\sigma}}{4}=\frac{1}{2}(\sigma G+G \sigma) \\
\Longleftrightarrow & G=L_{\sigma}[x \dot{\sigma}+y \tilde{\sigma}], \quad 4 y=\operatorname{Tr}(G \tilde{\sigma})=\operatorname{Tr}\left(L_{\sigma}[x \dot{\sigma}+y \tilde{\sigma}] \tilde{\sigma}\right), \\
\Longleftrightarrow & x=4-\operatorname{Tr}\left(L_{\sigma}[\tilde{\sigma}] \tilde{\sigma}\right), \quad y=\operatorname{Tr}\left(L_{\sigma}[\dot{\sigma}] \tilde{\sigma}\right) .
\end{aligned}
$$

Note that in Eq. (E9) we have used $x \dot{\sigma}+y \tilde{\sigma}=\frac{1}{2}(G \sigma+\sigma G)$ and $\operatorname{Tr}\left(G^{2} \sigma\right)=\operatorname{Tr}[G(x \dot{\sigma}+y \tilde{\sigma})]$. Substituting the optimal $G=L_{\sigma}[x \dot{\sigma}+y \tilde{\sigma}]$ into Eq. (E6) where $x, y$ satisfy Eq. (E10), we obtain

$$
\mathfrak{F}_{\mathrm{SQL}}\left(\mathcal{D}_{L, \omega}\right)=\operatorname{Tr}\left(L_{\sigma}[\dot{\sigma}] \dot{\sigma}\right)+\frac{\operatorname{Tr}\left(L_{\sigma}[\dot{\sigma}] \tilde{\sigma}\right)^{2}}{4-\operatorname{Tr}\left(L_{\sigma}[\tilde{\sigma}] \tilde{\sigma}\right)}+O(\varepsilon)
$$

shown as Eq. (41) in the main text. 
Next we express $\operatorname{Tr}\left(L_{\sigma}[\dot{\sigma}] \dot{\sigma}\right), \operatorname{Tr}\left(L_{\sigma}[\dot{\sigma}] \tilde{\sigma}\right)$, and $\operatorname{Tr}\left(L_{\sigma}[\tilde{\sigma}] \tilde{\sigma}\right)$ in terms of $C$ and $\tilde{C}$. Let $\tau=E^{\dagger} E, \tilde{\tau}=E^{\dagger} F+F^{\dagger} E, \tau^{\prime}=$ $i E^{\dagger} \dot{E}-i \dot{E}^{\dagger} E$ such that

$$
\begin{gathered}
\tau_{i j}=\operatorname{Tr}\left(C^{\dagger} K_{i}^{\dagger} K_{j} C\right), \quad \tilde{\tau}_{i j}=\operatorname{Tr}\left(\tilde{C} K_{i}^{\dagger} K_{j}\right), \\
\tau_{i j}^{\prime}=i \operatorname{Tr}\left(C^{\dagger} K_{i}^{\dagger} \dot{K}_{j} C\right)-i \operatorname{Tr}\left(C^{\dagger} \dot{K}_{i}^{\dagger} K_{j} C\right) .
\end{gathered}
$$

Without loss of generality, assume that $\tau_{i j}=\operatorname{Tr}\left(C^{\dagger} K_{i}^{\dagger} K_{j} C\right)=\lambda_{i} \delta_{i j}$, which could always be achieved by performing a unitary transformation on $\mathbf{K}$. We also have $\lambda_{i}>0$ for all $i$ because $C$ is full rank and the $\left\{\left|K_{i}\right\rangle\right\}_{i=1}^{r}$ are linearly independent. Using an orthonormal basis $\{|i\rangle\rangle\}_{i=1}^{d^{2}}$, where $\left.\left.|i\rangle\right\rangle=\left(1 / \sqrt{\lambda_{i}}\right)\left|K_{i} C\right\rangle\right\rangle$ for $1 \leq i \leq r$, we have

$$
\begin{aligned}
& \sigma=\left(\begin{array}{cc}
\left(\lambda_{i} \delta_{i j}\right) & 0 \\
0 & 0
\end{array}\right), \\
& \dot{\sigma}=\left(\begin{array}{cc}
\left(\left\langle\left\langle K_{i} C \mid \dot{K}_{j} C\right\rangle\right\rangle \sqrt{\frac{\lambda_{j}}{\lambda_{i}}}+\sqrt{\frac{\lambda_{i}}{\lambda_{j}}}\left\langle\left\langle\dot{K}_{i} C \mid K_{j} C\right\rangle\right\rangle\right) & \left(\left\langle\left\langle\dot{K}_{i} C \mid j^{\prime}\right\rangle\right\rangle \sqrt{\lambda_{i}}\right) \\
\left(\left\langle\left\langle i^{\prime} \mid \dot{K}_{j} C\right\rangle\right\rangle \sqrt{\lambda_{j}}\right) & 0
\end{array}\right), \\
& \tilde{\sigma}=\left(\begin{array}{cc}
\left(i\left\langle\left\langle K_{i} C \mid K_{j} D\right\rangle\right\rangle \sqrt{\frac{\lambda_{j}}{\lambda_{i}}}-i \sqrt{\frac{\lambda_{i}}{\lambda_{j}}}\left\langle\left\langle K_{i} D \mid K_{j} C\right\rangle\right\rangle\right) & \left(-i\left\langle\left\langle K_{i} D \mid j^{\prime}\right\rangle\right\rangle \sqrt{\lambda_{i}}\right) \\
\left(i\left\langle\left\langle i^{\prime} \mid K_{j} D\right\rangle\right\rangle \sqrt{\lambda_{j}}\right) & 0
\end{array}\right),
\end{aligned}
$$

where $1 \leq i, j \leq r$ and $r+1 \leq i^{\prime}, j^{\prime} \leq d^{2}$. Then we can show Eqs. (44)-(46) in the main text:

$$
\begin{aligned}
\operatorname{Tr}\left(L_{\sigma}[\dot{\sigma}] \dot{\sigma}\right) & =2 \sum_{i, j} \frac{\left|(\dot{\sigma})_{i j}\right|^{2}+\lambda_{j}>0}{\lambda_{i}+\lambda_{j}} \\
& =2 \sum_{i, j=1}^{r} \frac{\left|\left\langle\left\langle K_{i} C \mid \dot{K}_{j} C\right\rangle\right\rangle \sqrt{\lambda_{j} / \lambda_{i}}+\left(\sqrt{\lambda_{i} / \lambda_{j}}\right)\left\langle\left\langle\dot{K}_{i} C \mid K_{j} C\right\rangle\right\rangle\right|^{2}}{\lambda_{i}+\lambda_{j}}+4 \sum_{i^{\prime}=r+1}^{d^{2}} \sum_{j=1}^{r} \frac{\left.\left|\left\langle i^{\prime} \mid \dot{K}_{j} C\right\rangle\right\rangle \sqrt{\lambda_{j}}\right|^{2}}{\lambda_{j}} \\
& =4 \operatorname{Tr}\left(C^{\dagger} \dot{K}^{\dagger} \dot{\mathbf{K}} C\right)+2 \sum_{i, j=1}^{r} \frac{\left|\left\langle\left\langle K_{i} C \mid \dot{K}_{j} C\right\rangle\right\rangle \sqrt{\lambda_{j} / \lambda_{i}}+\left(\sqrt{\lambda_{i} / \lambda_{j}}\right)\left\langle\left\langle\dot{K}_{i} C \mid K_{j} C\right\rangle\right\rangle\right|^{2}}{\lambda_{i}+\lambda_{j}}-2 \frac{\left|\left\langle\left\langle K_{i} C \mid \dot{K}_{j} C\right\rangle\right\rangle\right|^{2}}{\lambda_{i}} \\
& =4 \operatorname{Tr}\left(C^{\dagger} \dot{\mathbf{K}}^{\dagger} \dot{\mathbf{K}} C\right)-2 \sum_{i, j=1}^{r} \frac{\left|\tau_{i j}^{\prime}\right|^{2}}{\lambda_{i}+\lambda_{j}} \\
& =4 \operatorname{Tr}\left(C^{\dagger} \dot{\mathbf{K}}^{\dagger} \dot{\mathbf{K}} C\right)-\operatorname{Tr}\left(L_{\tau}\left[\tau^{\prime}\right] \tau^{\prime}\right),
\end{aligned}
$$

$$
\begin{aligned}
\operatorname{Tr}\left(L_{\sigma}[\tilde{\sigma}] \tilde{\sigma}\right) & =2 \sum_{i, j: \lambda_{i}+\lambda_{j}>0} \frac{\left|(\tilde{\sigma})_{i j}\right|^{2}}{\lambda_{i}+\lambda_{j}} \\
& =2 \sum_{i, j=1}^{r} \frac{\left|i\left\langle\left\langle K_{i} C \mid K_{j} D\right\rangle\right\rangle \sqrt{\lambda_{j} / \lambda_{i}}-i\left(\sqrt{\lambda_{i} / \lambda_{j}}\right)\left\langle\left\langle K_{i} D \mid K_{j} C\right\rangle\right\rangle\right|^{2}}{\lambda_{i}+\lambda_{j}}+4 \sum_{i^{\prime}=r+1}^{d^{2}} \sum_{j=1}^{r} \frac{\left.\left|i\left\langle i^{\prime} \mid K_{j} D\right\rangle\right\rangle \sqrt{\lambda_{j}}\right|^{2}}{\lambda_{j}}
\end{aligned}
$$




$$
\begin{aligned}
& =4+2 \sum_{i, j=1}^{r} \frac{\left|i\left\langle\left\langle K_{i} C \mid K_{j} D\right\rangle\right\rangle \sqrt{\lambda_{j} / \lambda_{i}}-i\left(\sqrt{\lambda_{i} / \lambda_{j}}\right)\left\langle\left\langle K_{i} D \mid K_{j} C\right\rangle\right\rangle\right|^{2}}{\lambda_{i}+\lambda_{j}}-2 \frac{\left|\left\langle\left\langle K_{i} C \mid K_{j} D\right\rangle\right\rangle\right|^{2}}{\lambda_{i}} \\
& =4-2 \sum_{i j} \frac{\left|\tilde{\tau}_{i j}\right|^{2}}{\lambda_{i}+\lambda_{j}} \\
& =4-\operatorname{Tr}\left(L_{\tau}[\tilde{\tau}] \tilde{\tau}\right),
\end{aligned}
$$

and

$$
\begin{aligned}
\operatorname{Tr}\left(L_{\sigma}[\dot{\sigma}] \tilde{\sigma}\right) & =2 \sum_{i, j: \lambda_{i}+\lambda_{j} \neq 0} \frac{\dot{\sigma}_{i j} \tilde{\sigma}_{j i}}{\lambda_{i}+\lambda_{j}} \\
& =2 \sum_{i, j=1}^{r} \frac{\dot{\sigma}_{i j} \tilde{\sigma}_{j i}}{\lambda_{i}+\lambda_{j}}+2 \sum_{i^{\prime}=r+1}^{d^{2}} \sum_{j=1}^{r} \frac{\dot{\sigma}_{i^{\prime} j} \tilde{\sigma}_{j i^{\prime}}}{\lambda_{j}}+2 \sum_{i^{\prime}=r+1}^{d^{2}} \sum_{j=1}^{r} \frac{\dot{\sigma}_{j i^{\prime}} \tilde{\sigma}_{i^{\prime} j}}{\lambda_{j}} \\
& =-2 \operatorname{Tr}(\tilde{C} H)+2 \sum_{i, j=1}^{r} \frac{\dot{\sigma}_{i j} \tilde{\sigma}_{j i}}{\lambda_{i}+\lambda_{j}}+2 i \sum_{i, j=1}^{r} \frac{\left\langle\left\langle K_{j} D \mid K_{i} C\right\rangle\right\rangle\left\langle\left\langle K_{i} C \mid \dot{K}_{j} C\right\rangle\right\rangle}{\lambda_{i}}-\frac{\left\langle\left\langle\dot{K}_{j} C \mid K_{i} C\right\rangle\right\rangle\left\langle\left\langle K_{i} C \mid K_{j} D\right\rangle\right\rangle}{\lambda_{i}} \\
& =-2 \operatorname{Tr}(\tilde{C} H)+2 \sum_{i, j=1}^{r} \frac{\tau_{i j}^{\prime} \tilde{\tau}_{j i}}{\lambda_{i}+\lambda_{j}} \\
& =-2 \operatorname{Tr}(\tilde{C} H)+\operatorname{Tr}\left(L_{\tau}\left[\tau^{\prime}\right] \tilde{\tau}\right) .
\end{aligned}
$$

Therefore, we conclude that

$$
\begin{aligned}
\mathfrak{F}_{\mathrm{SQL}}\left(\mathcal{D}_{L, \omega}\right) & \approx f(C, \tilde{C}) \\
& =4 \operatorname{Tr}\left(C^{\dagger} \dot{\mathbf{K}}^{\dagger} \dot{\mathbf{K}} C\right)-\operatorname{Tr}\left(L_{\tau}\left[\tau^{\prime}\right] \tau^{\prime}\right)+\frac{\left[-2 \operatorname{Tr}(\tilde{C} H)+\operatorname{Tr}\left(L_{\tau}\left[\tau^{\prime}\right] \tilde{\tau}\right)\right]^{2}}{\operatorname{Tr}\left(L_{\tau}[\tilde{\tau}] \tilde{\tau}\right)} .
\end{aligned}
$$

Next, we want to show that

$$
\max _{\tilde{C} \in \mathbb{H}_{d}} f(C, \tilde{C})=\min _{h: \beta=0} 4 \operatorname{Tr}\left(C^{\dagger} \alpha C\right)
$$

when $C$ is full rank. To calculate the dual program of the right-hand side, we introduce a Hermitian matrix $\tilde{C}$ as a Lagrange multiplier of $\beta=0$ [98]. The Lagrange function is

$$
L(\tilde{C}, h)=4 \operatorname{Tr}\left[C^{\dagger}(\dot{\mathbf{K}}-i h \mathbf{K})^{\dagger}(\dot{\mathbf{K}}-i h \mathbf{K}) C\right]+\operatorname{Tr}\left[\tilde{C}\left(H+\mathbf{K}^{\dagger} h \mathbf{K}\right)\right] .
$$

Then

$$
\begin{aligned}
\min _{h} L(\tilde{C}, h) & =\min _{h} 4 \operatorname{Tr}\left[C^{\dagger}(\dot{\mathbf{K}}-i h \mathbf{K})^{\dagger}(\dot{\mathbf{K}}-i h \mathbf{K}) C\right]+\operatorname{Tr}\left[\tilde{C}\left(H+\mathbf{K}^{\dagger} h \mathbf{K}\right)\right] \\
& =\min _{h} 4 \operatorname{Tr}\left(C^{\dagger} \dot{\mathbf{K}}^{\dagger} \dot{\mathbf{K}} C\right)+4 \operatorname{Tr}\left(\tau h^{2}\right)+4 \operatorname{Tr}\left(i C^{\dagger} \mathbf{K}^{\dagger} h \dot{\mathbf{K}} C-i C^{\dagger} \dot{K}^{\dagger} h \mathbf{K} C\right)+\operatorname{Tr}\left[\tilde{C}\left(H+\mathbf{K}^{\dagger} h \mathbf{K}\right)\right] \\
& =\min _{h} 4 \operatorname{Tr}\left(C^{\dagger} \dot{\mathbf{K}}^{\dagger} \dot{\mathbf{K}} C\right)+4 \operatorname{Tr}\left(\tau h^{2}\right)+4 \operatorname{Tr}\left(h^{T} \tau^{\prime}\right)+\operatorname{Tr}(\tilde{C} H)+\operatorname{Tr}\left(h^{T} \tilde{\tau}\right) \\
& =4 \operatorname{Tr}\left(C^{\dagger} \dot{\mathbf{K}}^{\dagger} \dot{\mathbf{K}} C\right)+\operatorname{Tr}(\tilde{C} H)-\frac{1}{8} \sum_{i, j=1}^{r} \frac{\left|4 \tau_{i j}^{\prime}+\tilde{\tau}_{i j}\right|^{2}}{\lambda_{i}+\lambda_{j}} .
\end{aligned}
$$


The dual program is

$$
\begin{aligned}
\max _{\tilde{C}} \min _{h} L(\tilde{C}, h) & =\max _{\tilde{C}} 4 \operatorname{Tr}\left(C^{\dagger} \dot{\mathbf{K}}^{\dagger} \dot{\mathbf{K}} C\right)+\operatorname{Tr}(\tilde{C} H)-\frac{1}{8} \sum_{i, j=1}^{r} \frac{16\left|\tau_{i j}^{\prime}\right|^{2}+\left|\tilde{\tau}_{i j}\right|^{2}+4\left(\tilde{\tau}_{i j} \tau_{j i}^{\prime}+\tilde{\tau}_{j i} \tau_{i j}^{\prime}\right)}{\lambda_{i}+\lambda_{j}} \\
& =\max _{\tilde{C}, x} 4 \operatorname{Tr}\left(C^{\dagger} \dot{\mathbf{K}}^{\dagger} \dot{\mathbf{K}} C\right)+x \operatorname{Tr}(\tilde{C} H)-\frac{1}{8} \sum_{i, j=1}^{r} \frac{16\left|\tau_{i j}^{\prime}\right|^{2}+x^{2}\left|\tilde{\tau}_{i j}\right|^{2}+8 x \tilde{\tau}_{i j} \tau_{j i}^{\prime}}{\lambda_{i}+\lambda_{j}} \\
& =\max _{\tilde{C}} 4 \operatorname{Tr}\left(C^{\dagger} \dot{\mathbf{K}}^{\dagger} \dot{\mathbf{K}} C\right)-2 \sum_{i, j=1}^{r} \frac{\left|\tau_{i j}^{\prime}\right|^{2}}{\lambda_{i}+\lambda_{j}}+\frac{\left[-\operatorname{Tr}(\tilde{C} H)+\sum_{i, j=1}^{r} \tilde{\tau}_{i j} \tau_{j i}^{\prime} /\left(\lambda_{i}+\lambda_{j}\right)\right]^{2}}{(1 / 2) \sum_{i, j=1}^{r}\left|\tilde{\tau}_{i j}\right|^{2} /\left(\lambda_{i}+\lambda_{j}\right)} \\
& =\max _{\tilde{C}} f(C, \tilde{C}),
\end{aligned}
$$

where we have used the fact that $\tilde{C} \leftarrow x \tilde{C}$ does not change the result. Equation (E21) is then proved.

Moreover, the optimal $\tilde{C}$ in Eq. (E21) must be traceless. Suppose that $\tilde{C}$ is optimal in Eq. (E21). We prove that $\operatorname{Tr}(\tilde{C})=$ 0 . Let $z$ be a real number. Then

$$
q(z):=f\left(C, \tilde{C}+z C C^{\dagger}\right)=\frac{s(z)^{2}}{t(z)}+\text { const. }
$$

Since $\max _{z} q(z)=q(0)$, we have $q^{\prime}(0)=s(0)\left[2 s^{\prime}(0) t(0)-s(0) t^{\prime}(0)\right] / t(0)^{2}=0$. We have

$$
\begin{aligned}
& s(z)=-\operatorname{Tr}\left[\left(\tilde{C}+z C C^{\dagger}\right) H\right]+\sum_{i, j=1}^{r} \frac{\left(\tilde{\tau}_{i j}+z \lambda_{i} \delta_{i j}\right) \tau_{i j}^{\prime}}{\lambda_{i}+\lambda_{j}}, \\
& s^{\prime}(0)=-\operatorname{Tr}\left(C C^{\dagger} H\right)+\sum_{i=1}^{r} \frac{1}{2} \tau_{i i}^{\prime}=0, \\
& t(z)=\frac{1}{2} \sum_{i, j=1}^{r} \frac{\left|\tilde{\tau}_{i j}+z \lambda_{i} \delta_{i j}\right|^{2}}{\lambda_{i}+\lambda_{j}}=\frac{1}{2} \sum_{i, j=1}^{r} \frac{\left|\tilde{\tau}_{i j}\right|^{2}+z \lambda_{i} \delta_{i j}\left(\tilde{\tau}_{i j}^{*}+\tilde{\tau}_{i j}\right)+z^{2} \lambda_{i}^{2} \delta_{i j}}{\lambda_{i}+\lambda_{j}}, \\
& t^{\prime}(0)=\frac{1}{2} \sum_{i, j=1}^{r} \frac{\lambda_{i} \delta_{i j}\left(\tilde{\tau}_{i j}^{*}+\tilde{\tau}_{i j}\right)}{\lambda_{i}+\lambda_{j}}=\frac{1}{2} \sum_{i=1}^{r} \tilde{\tau}_{i i}=\frac{1}{2} \operatorname{Tr}(\tilde{C}) .
\end{aligned}
$$

Then $q^{\prime}(0)=0$ implies that $\operatorname{Tr}(\tilde{C})=0$.

\section{APPENDIX F: NUMERICAL ALGORITHM TO FIND THE OPTIMAL CODE WHEN THE HNKS CONDITION IS VIOLATED}

\section{Finding the optimal $C$}

We first describe a numerical algorithm finding a full rank $C^{\diamond}$ such that $\operatorname{Tr}\left(C^{\diamond \dagger} C^{\diamond}\right)=1$ and

$$
\min _{h: \beta=0} 4 \operatorname{Tr}\left(C^{\diamond \dagger} \alpha C^{\diamond}\right)>\mathfrak{F}_{\mathrm{SQL}}^{(u)}\left(\mathcal{E}_{\omega}\right)-\eta / 2
$$

for any $\eta>0$. We first note that $\mathfrak{F}_{\mathrm{SQL}}^{(u)}\left(\mathcal{E}_{\omega}\right)=\min _{h: \beta=0} 4$ $\|\alpha\|$ can be calculated via the SDP [28] $\min _{h} x \quad$ subject to $\left(\begin{array}{cccc}x I_{d} & \tilde{K}_{1}^{\dagger} & \cdots & \tilde{K}_{r}^{\dagger} \\ \tilde{K}_{1} & I_{d^{\prime}} & \cdots & 0 \\ \vdots & 0 & \ddots & \vdots \\ \tilde{K}_{r} & 0 & \cdots & I_{d^{\prime}}\end{array}\right) \succeq 0, \quad \beta=0$,

where $d$ and $d^{\prime}$ are the input and output dimensions of $\mathcal{E}_{\omega}$, $I_{n}$ is a $n \times n$ identity matrix, and $\tilde{\mathbf{K}}=\dot{\mathbf{K}}-i h \mathbf{K}$.

To find the full rank $C^{\diamond}$, we first find a density matrix $\rho^{\diamond}$ such that

$$
\min _{h: \beta=0} 4 \operatorname{Tr}\left(\rho^{\diamond} \alpha\right)=\min _{h: \beta=0} 4\|\alpha\|
$$


This can be achieved via the following algorithm [52].

(i) Find an $h^{\diamond}$ using the SDP [Eq. (F2)] such that $\alpha^{\diamond}=$ $\left.\alpha\right|_{h=h^{\diamond}}$ satisfies $\left\|\alpha^{\diamond}\right\|=\min _{h: \beta=0}\|\alpha\|$.

(ii) Let $\Pi^{\diamond}$ be the projection onto the subspace spanned by all eigenstates corresponding to the largest eigenvalue of $\alpha^{\diamond}$. We find an optimal density matrix $\rho^{\diamond}$ satisfying $\Pi^{\diamond} \rho^{\diamond} \Pi^{\diamond}=\rho^{\diamond}$ and

$$
\begin{aligned}
& \operatorname{Re}\left\{\operatorname{Tr}\left[\rho^{\diamond}\left(i \mathbf{K}^{\dagger} \Delta h\right)\left(\dot{\mathbf{K}}-i h^{\diamond} \mathbf{K}\right)\right]\right\}=0 \\
& \quad \text { for all } \eta \in \mathbb{H}_{r} \text { such that } \mathbf{K}^{\dagger} \Delta h \mathbf{K}=0 .
\end{aligned}
$$

Then $C^{\diamond}=\left[\left(1-\eta^{\prime}\right) \rho^{\diamond}+\eta^{\prime} I / d\right]^{1 / 2}$, where $\eta^{\prime}=\eta /\left[2 \mathfrak{F}_{\mathrm{SQL}}^{(u)}\right.$ $\left.\left(\mathcal{E}_{\omega}\right)\right]$ is a full-rank matrix satisfying

$$
\begin{aligned}
& \min _{h: \beta=0} 4 \operatorname{Tr}\left(C^{\diamond \dagger} \alpha C^{\diamond}\right)>\left(1-\eta^{\prime}\right) \mathfrak{F}_{\mathrm{SQL}}^{(u)}\left(\mathcal{E}_{\omega}\right) \\
& \quad=\mathfrak{F}_{\mathrm{SQL}}^{(u)}\left(\mathcal{E}_{\omega}\right)-\eta / 2 .
\end{aligned}
$$

The algorithm above can also be used to find $\rho \star$, whose purification is the optimal input state of a single quantum channel $\mathcal{E}_{\omega}$ achieving $\mathfrak{F}_{1}\left(\mathcal{E}_{\omega}\right)$.

(i) Find an $h$ using the SDP in Eq. (F2) without the requirement that $\beta=0$, such that $\alpha=\left.\alpha\right|_{h=h} \bullet$ satisfies $\left\|\alpha \bullet=\min _{h}\right\| \alpha \|$.

(ii) Let $\Pi^{\star}$ be the projection onto the subspace spanned by all eigenstates corresponding to the largest eigenvalue of $\alpha^{\star}$. We find an optimal density matrix $\rho$ satisfying $\Pi^{\bullet} \rho \Pi^{\star}=\rho \bullet$ and

$$
\begin{aligned}
& \operatorname{Re}\left\{\operatorname{Tr}\left[\rho^{\diamond}\left(i \mathbf{K}^{\dagger} \Delta h\right)\left(\dot{\mathbf{K}}-i h^{\diamond} \mathbf{K}\right)\right]\right\}=0 \\
& \quad \text { for all } \Delta h \in \mathbb{H}_{r} .
\end{aligned}
$$

\section{Validity of the algorithm to find the optimal $C$}

For completeness, we prove the validity of the above algorithm. According to Sion's minimax theorem [96,97], for convex compact sets $\mathfrak{P} \subset \mathbb{R}^{m}$ and $\mathfrak{Q} \subset \mathbb{R}^{n}$ and $g: P \times$ $Q \rightarrow \mathbb{R}$ such that $g(x, y)$ is a continuous convex (concave) function in $x(y)$ for every fixed $y(x)$,

$$
\max _{y \in \mathfrak{Q}} \min _{x \in \mathfrak{P}} g(x, y)=\min _{x \in \mathfrak{P}} \max _{y \in \mathfrak{Q}} g(x, y) .
$$

In particular, if $\left(x^{\mathbf{\Delta}}, y^{\bullet}\right)$ is a solution of $\max _{y \in \mathfrak{Q}} \min _{x \in \mathfrak{P}}$ $g(x, y)$ then there must exist an $x \star$ such that $(x \bullet, y)$ is a saddle point. Let $\left(x^{\star}, y^{\mathbf{\Delta}}\right)$ be a solution of $\min _{x \in \mathfrak{P}} \max _{y \in \mathfrak{Q}} g(x, y)$. Then we must have

$$
g\left(x^{\mathbf{}}, y^{\bullet}\right) \leq g\left(x^{\star}, y^{\bullet}\right) \leq g\left(x^{\star}, y^{\mathbf{\Delta}}\right) .
$$

According to Eq. (F7), $g\left(x^{\mathbf{\Delta}}, y^{\bullet}\right)=g\left(x^{\star}, y^{\mathbf{\Delta}}\right)$ and all equalities must hold for the above equation. Moreover,

$g\left(x^{\Downarrow}, y\right) \leq g\left(x^{\Downarrow}, y^{\Downarrow}\right) \leq g(x, y \Downarrow)$ for all $(x, y) \in \mathfrak{P} \times \mathfrak{Q}$, which means that $(x, y)$ is a saddle point. For example, we can take $x=h \in \mathbb{H}_{r}, y=C C^{\dagger}=\rho \in \mathfrak{S}\left(\mathcal{H}_{\mathcal{P}}\right)$, and $g(x, y)=4 \operatorname{Tr}(\rho \alpha)$. (We can also add the constraint $\beta=0$ on $h$ that does not affect our discussion below.) Then the solution of the above optimization problem is $\mathfrak{F}_{1}\left(\mathcal{E}_{\omega}\right)$ [or $\mathfrak{F}_{\mathrm{SQL}}\left(\mathcal{E}_{\omega}\right)$ with the constraint $\left.\beta=0\right]$. Note that we can always confine $h$ in a compact set such that the solutions are not altered and the minimax theorem is applicable [52]. Let $\left(h^{\mathbf{\Lambda}}, \rho^{\boldsymbol{}}\right)$ be any solution of the optimization problem $\max _{\rho} \min _{h} 4 \operatorname{Tr}(\rho \alpha)$. Then there exists an $h$ such that $\left(h^{\star}, \rho^{\star}\right)$ is a saddle point. Similarly, if $g\left(x^{\star}, y^{\star}\right)$ is a solution of $\min _{x \in \mathfrak{P}} \max _{y \in \mathfrak{Q}} g(x, y)$, which in our case is a SDP [Eq. (F2)], there must exist a $y^{\star}$ such that $\left(x^{\star}, y^{\star}\right)$ is a saddle point. Let $\left(h^{\star}, \rho^{\mathbf{\Delta}}\right)$ be any solution of the optimization problem $\min _{h} \max _{\rho} 4 \operatorname{Tr}(\rho \alpha)$. Then there exists an $\rho$ such that $\left(h^{\star}, \rho^{\star}\right)$ is a saddle point. Moreover, $\left(h^{\star}, \rho\right)$ is a saddle point if and only if

(i) $\operatorname{Tr}\left(\rho^{\star}\right)=\|\alpha\|$ if and only if $\operatorname{Tr}\left(\rho^{\star} \alpha^{\star}\right) \geq$ $\operatorname{Tr}(\rho \alpha)$ for all $\rho$,

(ii) $\operatorname{Re}\left\{\operatorname{Tr}\left[\rho^{\bullet}\left(i \mathbf{K}^{\dagger} \Delta h\right)\left(\dot{\mathbf{K}}-i h^{\bullet} \mathbf{K}\right)\right]\right\}=0$ for all $\Delta h \in \mathbb{H}_{r}$ if and only if $\operatorname{Tr}\left(\rho{ }^{\star}\right) \leq \operatorname{Tr}(\rho \star \alpha)$ for all $h$.

This justifies the validity of the algorithm we described above.

\section{Finding the optimal $\tilde{\boldsymbol{C}}$}

Next, we describe how to find $\tilde{C}^{\diamond}$ such that $f\left(C^{\diamond}, \tilde{C}^{\diamond}\right)=$ $\max _{\tilde{C}} f\left(C^{\diamond}, \tilde{C}\right)=\min _{h: \beta=0} 4 \operatorname{Tr}\left(C^{\diamond \dagger} \alpha C^{\diamond}\right)$. According to Appendix E,

$$
\begin{aligned}
f(C, \tilde{C})= & 4 \operatorname{Tr}\left(C^{\dagger} \dot{\mathbf{K}}^{\dagger} \dot{\mathbf{K}} C\right)-2 \sum_{i, j=1}^{r} \frac{\left|\tau_{i j}^{\prime}\right|^{2}}{\lambda_{i}+\lambda_{j}} \\
& +\frac{\left[-\operatorname{Tr}(\tilde{C} H)+\sum_{i, j=1}^{r} \tilde{\tau}_{i j} \tau_{j i}^{\prime} /\left(\lambda_{i}+\lambda_{j}\right)\right]^{2}}{\frac{1}{2} \sum_{i, j=1}^{r}\left|\tilde{\tau}_{i j}\right|^{2} /\left(\lambda_{i}+\lambda_{j}\right)},
\end{aligned}
$$

where we have assumed that $\tau_{i j}=\operatorname{Tr}\left(C^{\dagger} K_{i}^{\dagger} K_{j} C\right)=\lambda_{i} \delta_{i j}$. For a fixed $C, \tilde{\tau}$ is a linear function in $\tilde{C}$. We can always write

$$
f(C, \tilde{C})=f_{1}(C)+\frac{\left.\left|\left\langle\tilde{C} \mid f_{2}(C)\right\rangle\right\rangle\right|^{2}}{\left\langle\left\langle\tilde{C}\left|f_{3}(C)\right| \tilde{C}\right\rangle\right\rangle},
$$

where $f_{1}(C) \in \mathbb{R}, f_{2}(C) \in \mathbb{C}^{d \times d}$ is Hermitian, and $f_{3}(C) \in$ $\mathbb{C}^{d^{2} \times d^{2}}$ is positive semidefinite. Moreover, $\left.\left|f_{2}(C)\right\rangle\right\rangle$ is in the support of $f_{3}(C)$. The $f_{1,2,3}(C)$ are functions of $C$ only. According to the Cauchy-Schwarz inequality,

$$
\max _{\tilde{C}} f(C, \tilde{C})=f_{1}(C)+\left\langle\left\langle f_{2}(C)\left|f_{3}(C)^{-1}\right| f_{2}(C)\right\rangle\right\rangle
$$

with the maximum attained when $\left.|\tilde{C}\rangle\rangle=f_{3}(C)^{-1}\left|f_{2}(C)\right\rangle\right\rangle$, where the superscript " -1 " here means the Moore-Penrose 
pseudoinverse. Therefore, we take

$$
\left.\left.\left|\tilde{C}^{\diamond}\right\rangle\right\rangle=f_{3}\left(C^{\diamond}\right)^{-1}\left|f_{2}\left(C^{\diamond}\right)\right\rangle\right\rangle \text {. }
$$

\section{APPENDIX G: A SDP TO FIND THE OPTIMAL INPUT STATE OF A NOISY MACH-ZEHNDER INTERFEROMETER}

Here we consider a two-arm Mach-Zehnder interferometer with one noisy arm and one noiseless arm, where the input state is an $M$-photon state

$$
\left|\psi_{0}\right\rangle=\sum_{m=0}^{M} \gamma_{m}|m\rangle|M-m\rangle .
$$

Here $|m, M-m\rangle$ represents a two-mode Fock state where $m$ is the number of photons in the first mode and $M-$ $m$ is the number of photons in the second mode. The noisy quantum channel $\mathcal{E}_{\omega}(\cdot)$ acting on the first mode is described by the Kraus operators

$$
K_{i}=\sqrt{\frac{(1-p)^{i}}{i !}} e^{-i \omega \hat{a}^{\dagger} \hat{a}} p^{\hat{a}^{\dagger} \hat{a} / 2} \hat{a}^{i}, \quad i=0,1, \ldots, M,
$$

where $\omega$ is the unknown phase to be estimated, $\hat{a}$ is the photon annihilation operator, $0<p<1$ is the loss rate, and $K_{i}$ is associated with losing $i$ photons. Note that we are allowed to truncate the maximum photon number at $M$ because of the restriction on the input state [Eq. (G1)].

We show that the algorithm described in Appendix $\mathrm{F}$ naturally gives a SDP for obtaining the optimal $\left\{\gamma_{m}\right\}_{m=0}^{M}$. We emphasize here that it was already shown in Ref. [82] that solving for the optimal input state in an interferometer with two noisy arms is a convex optimization problem. Here we provide an alternative algorithm as a demonstration of our approach that also contains a proof that states of form (G1) are optimal for $\mathcal{E}_{\omega}$.

Recall that, given $\mathcal{E}_{\omega}$, we can find an optimal input state achieving $\mathfrak{F}_{1}\left(\mathcal{E}_{\omega}\right)$ by purifying $\rho$, which is a solution of

$$
\mathfrak{F}_{1}\left(\mathcal{E}_{\omega}\right)=\max _{\rho} \min _{h} 4 \operatorname{Tr}(\rho \alpha)=\min _{h} 4\|\alpha\| .
$$

We show that the optimization problem above has a diagonal solution of $\rho$. Note that

$$
\begin{aligned}
\alpha= & \sum_{i=0}^{M}\left(\dot{K}_{i}-i \sum_{i, j=0}^{M} h_{i j} K_{j}\right)^{\dagger}\left(\dot{K}_{i}-i \sum_{j^{\prime}=0}^{M} h_{i j^{\prime}} K_{j^{\prime}}\right) \\
= & \sum_{i=0}^{M}\left(\dot{K}_{i}-i h_{i i} K_{i}\right)^{\dagger}\left(\dot{K}_{i}-i h_{i i} K_{i}\right) \\
& +\sum_{i=0}^{M} \sum_{j \neq i} K_{j}^{\dagger} h_{i j}^{*} h_{i j} K_{j}+\text { off-diagonal terms },
\end{aligned}
$$

where we divided $\alpha$ into diagonal terms and off-diagonal terms (in the Fork basis). The second term is always nonnegative and the off-diagonal terms will only increase $\|\alpha\|$. It is then clear that we can always assume that the optimal $h$ and the corresponding $\alpha$ are diagonal because

$$
\|\alpha\| \geq\left\|\sum_{i=0}^{M}\left(\dot{K}_{i}-i h_{i i} K_{i}\right)^{\dagger}\left(\dot{K}_{i}-i h_{i i} K_{j}\right)\right\| .
$$

Choose a diagonal $h^{\star}$, and let $\Pi^{\star}$ be the projection onto the subspace spanned by all eigenstates corresponding to the largest eigenvalue of $\alpha^{\star} ; \rho^{\star}$ is optimal if it satisfies $\Pi^{\bullet} \rho^{\bullet} \Pi^{\star}=\rho \bullet$ and

$\operatorname{Re}\left\{\operatorname{Tr}\left[\rho \rho^{\bullet}\left(i \mathbf{K}^{\dagger} \Delta h\right)\left(\dot{\mathbf{K}}-i h^{\bullet} \mathbf{K}\right)\right]\right\}=0 \quad$ for all $\Delta h \in \mathbb{H}_{M+1}$.

We observe that, when $\rho$ is optimal, the equation above still holds by replacing $\rho$ with its diagonal part. Then any diagonal $\rho$ that satisfies

$$
\begin{aligned}
& \operatorname{Re}\left\{\operatorname{Tr}\left[\rho \rho^{\diamond} K_{i}^{\dagger} \Delta h_{i i}\left(\dot{K}_{i}-i h_{i i}^{\diamond} K_{i}\right)\right]\right\}=0 \\
& \quad \text { for all }\left\{\Delta h_{i i}\right\}_{i=0}^{M} \in \mathbb{R}^{M+1}
\end{aligned}
$$

is optimal. Therefore, we can always assume that the input state has form (G1). Moreover, by assuming that $h$ and $\rho$ are diagonal, we only need to deal with diagonal operators in this algorithm and the problem is essentially a quadratically constrained quadratic program, which might admit more efficient numerical methods than the SDP formulation.

\section{APPENDIX H: CHANNEL QFI FOR THE SINGLE-QUBIT DEPOLARIZING CHANNELS}

Here we calculate $\mathfrak{F}_{1}, \mathfrak{F}_{\mathrm{SQL}}$, and $\mathfrak{F}_{\mathrm{HL}}$ for depolarizing channels

$$
\begin{aligned}
\mathcal{N}_{\omega}^{d}(\rho)= & (1-p) e^{-i \omega \sigma_{z} / 2} \rho e^{i \omega \sigma_{z} / 2}+p_{x} \sigma_{x} e^{-i \omega \sigma_{z} / 2} \rho e^{i \omega \sigma_{z} / 2} \sigma_{x} \\
& +p_{y} \sigma_{y} e^{-i \omega \sigma_{z} / 2} \rho e^{i \omega \sigma_{z} / 2} \sigma_{y} \\
& +p_{z} \sigma_{z} e^{-i \omega \sigma_{z} / 2} \rho e^{i \omega \sigma_{z} / 2} \sigma_{z}=\sum_{i=1}^{4} K_{i} \rho K_{i}^{\dagger}, \quad \text { (H1) }
\end{aligned}
$$

where $K_{1}=\sqrt{1-p} e^{-i \omega \sigma_{z} / 2}, K_{2}=\sqrt{p_{x}} \sigma_{x} e^{-i \omega \sigma_{z} / 2}, K_{3}=$ $\sqrt{p_{y}} \sigma_{y} e^{-i \omega \sigma_{z} / 2}$, and $K_{4}=\sqrt{p_{z}} \sigma_{z} e^{-i \omega \sigma_{z} / 2}$. We have

$$
\mathbf{K}=\left(\begin{array}{c}
\sqrt{1-p} \\
\sqrt{p_{x}} \sigma_{x} \\
\sqrt{p_{y}} \sigma_{y} \\
\sqrt{p_{z}} \sigma_{z}
\end{array}\right) e^{-i \omega \sigma_{z} / 2}, \quad \dot{\mathbf{K}}=\left(\begin{array}{c}
-\frac{i}{2} \sqrt{1-p} \sigma_{z} \\
-\frac{1}{2} \sqrt{p_{x}} \sigma_{y} \\
\frac{1}{2} \sqrt{p_{y}} \sigma_{x} \\
-\frac{i}{2} \sqrt{p_{z}}
\end{array}\right) e^{-i \omega \sigma_{z} / 2},
$$




$$
\beta=i \mathbf{K}^{\dagger}(\dot{\mathbf{K}}-i h \mathbf{K})=\frac{1}{2} \sigma_{z}+\mathbf{K}^{\dagger} h \mathbf{K},
$$

where

$$
\begin{aligned}
\beta=0 \Longrightarrow & (1-p) h_{11}+p_{x} h_{22}+p_{y} h_{33}+p_{z} h_{44}=0, \\
& \sqrt{(1-p) p_{x}}\left(h_{12}+h_{21}\right)+i \sqrt{p_{y} p_{z}} h_{34}-i \sqrt{p_{y} p_{z}} h_{43}=0, \\
& \sqrt{(1-p) p_{y}}\left(h_{13}+h_{31}\right)-i \sqrt{p_{x} p_{z}} h_{24}+i \sqrt{p_{x} p_{z}} h_{42}=0, \\
& \frac{1}{2}+\sqrt{(1-p) p_{z}}\left(h_{14}+h_{41}\right)+i \sqrt{p_{x} p_{y}} h_{23}-i \sqrt{p_{x} p_{y}} h_{32}=0 .
\end{aligned}
$$

Clearly, the HNKS condition is satisfied if and only if $p_{x}=$ $p_{z}=0$ or $p_{y}=p_{z}=0$. It is easy to see that, when $h_{i j}=0$ for all $i, j$ except $h_{23}, h_{32}, h_{14}$, and $h_{41}, \alpha=\|\alpha\| I,\|\alpha\|$ takes its minimum and

$$
\begin{aligned}
\|\alpha\|= & \frac{1}{4}+\sqrt{(1-p) p_{z}}\left(h_{14}+h_{41}\right)+i \sqrt{p_{x} p_{y}}\left(h_{23}-h_{32}\right) \\
& +\left(1-p+p_{z}\right)\left|h_{14}\right|^{2}+\left(p_{x}+p_{y}\right)\left|h_{23}\right|^{2} .
\end{aligned}
$$

Then

$$
\mathfrak{F}_{1}\left(\mathcal{N}_{\omega}^{d}\right)=4 \min _{h}\|\alpha\|=1-4\left(\frac{p_{x} p_{y}}{p_{x}+p_{y}}+\frac{(1-p) p_{z}}{1-p+p_{z}}\right) .
$$

When the HNKS condition is satisfied,

$$
\mathfrak{F}_{\mathrm{HL}}\left(\mathcal{N}_{\omega}^{d}\right)=4 \min _{h}\|\beta\|^{2}=1,
$$

and when the HNKS condition is violated,

$$
\begin{aligned}
& \mathfrak{F}_{\mathrm{SQL}}\left(\mathcal{N}_{\omega}^{d}\right)=4 \min _{h: \beta=0}\|\alpha\| \\
& \quad=-1+\frac{1}{4}\left(\frac{p_{x} p_{y}}{p_{x}+p_{y}}+\frac{(1-p) p_{z}}{1-p+p_{z}}\right)^{-1} .
\end{aligned}
$$

\section{APPENDIX I: SOLVING THE OPTIMAL QEC CODE FOR AMPLITUDE DAMPING CHANNELS}

In this appendix, we use the algorithm in Appendix $F$ to solve for the optimal QEC protocol analytically for amplitude damping channels with two Kraus operators:

$$
\begin{aligned}
\mathbf{K} & =\left(\begin{array}{c}
|0\rangle\langle 0|+\sqrt{1-p}| 1\rangle\langle 1| \\
\sqrt{p}|0\rangle\langle 1|
\end{array}\right) e^{-i \omega \sigma_{z} / 2} \\
& =\left(\begin{array}{c}
|0\rangle\left\langle 0\left|e^{-i \omega / 2}+\sqrt{1-p}\right| 1\right\rangle\langle 1| e^{i \omega / 2} \\
\sqrt{p}|0\rangle\langle 1| e^{i \omega / 2}
\end{array}\right) .
\end{aligned}
$$

Clearly, $H=i \mathbf{K}^{\dagger} \dot{\mathbf{K}}=\sigma_{z} / 2$.

\section{Finding the optimal $C$}

First, we want to find a full-rank normalized $C^{\diamond}$ such that $\min _{h: \beta=0} 4 \operatorname{Tr}\left(C^{\diamond \dagger} \alpha C^{\diamond}\right)$ is close to $\mathfrak{F}_{\mathrm{SQL}}^{(u)}\left(\mathcal{E}_{\omega}\right)$ and we follow the algorithm described in Appendix F 1.

We first compute $\alpha$ and $\beta$. Note that

$$
\dot{\mathbf{K}}=\left(\begin{array}{c}
-\frac{i}{2}|0\rangle\left\langle 0\left|+\frac{i}{2} \sqrt{1-p}\right| 1\right\rangle\langle 1| \\
\frac{i}{2} \sqrt{p}|0\rangle\langle 1|
\end{array}\right) e^{-i \omega \sigma_{z} / 2} .
$$

We first observe that in order to make $\beta=i \mathbf{K}^{\dagger}(\dot{\mathbf{K}}-i h \mathbf{K})=0, h$ has to be diagonal and then

$$
\dot{\mathbf{K}}-i h \mathbf{K}=\left(\begin{array}{c}
\left(-\frac{i}{2}-i h_{11}\right)|0\rangle\left\langle 0\left|+\left(\frac{i}{2}-i h_{11}\right) \sqrt{1-p}\right| 1\right\rangle\langle 1| \\
\left(\frac{i}{2}-i h_{22}\right) \sqrt{p}|0\rangle\langle 1|
\end{array}\right) e^{-i \omega \sigma_{z} / 2} .
$$

We also assume that $\omega=0$ for simplicity. Then 


$$
\begin{aligned}
\beta & =i \mathbf{K}^{\dagger}(\dot{\mathbf{K}}-i h \mathbf{K}) \\
& =\left(\frac{1}{2}+h_{11}\right)|0\rangle\left\langle 0\left|+\left(-\frac{1}{2}+h_{11}\right)(1-p)\right| 1\right\rangle\left\langle 1\left|+\left(-\frac{1}{2}+h_{22}\right) p\right| 1\right\rangle\langle 1| \\
& =0 \\
& \Longrightarrow \quad h_{11}=-\frac{1}{2}, \quad h_{22}=\frac{2-p}{2 p} .
\end{aligned}
$$

Therefore, $\alpha=(\dot{\mathbf{K}}-i h \mathbf{K})^{\dagger}(\dot{\mathbf{K}}-i h \mathbf{K})=\left[(1-p)+(1-p)^{2} / p\right]|1\rangle\langle 1|=[(1-p) / p]| 1\rangle\langle 1|$.

Since there is only one solution of $h$ such that $\beta=0$, there is no need to solve $\min _{h: \beta=0}\|\alpha\|$ using a SDP and the only solution is $\alpha^{\diamond}=[(1-p) / p]|1\rangle\langle 1|$ and $\mathfrak{F}_{\mathrm{SQL}}\left(\mathcal{N}_{\omega}^{\mathrm{AD}}\right)=4(1-p) / p$. We could take $C^{\diamond}=\sin \delta|0\rangle\langle 0|+\cos \delta| 1\rangle\langle 1|$, where $\delta$ is small. Note that here we use the small constant $\delta$ instead of $\eta^{\prime}$ in Eq. (F5) for simplicity. They are related by $\eta^{\prime} / 2=\sin ^{2}(\delta)$.

\section{Finding the optimal $\tilde{C}$}

Next we find the optimal $\tilde{C}^{\diamond}$ that minimizes

$$
\frac{\left[-\operatorname{Tr}(\tilde{C} H)+\sum_{i, j=1}^{r} \tilde{\tau}_{i j} \tau_{j i}^{\prime} /\left(\lambda_{i}+\lambda_{j}\right)\right]^{2}}{(1 / 2) \sum_{i, j=1}^{r}\left|\tilde{\tau}_{i j}\right|^{2} /\left(\lambda_{i}+\lambda_{j}\right)}=\frac{\left.\left|\left\langle\tilde{C} \mid f_{2}(C)\right\rangle\right\rangle\right|^{2}}{\left\langle\left\langle\tilde{C}\left|f_{3}(C)\right| \tilde{C}\right\rangle\right\rangle},
$$

and the solution is $\left.\left.\left|\tilde{C}^{\diamond}\right\rangle\right\rangle=f_{3}\left(C^{\diamond}\right)^{-1}\left|f_{2}\left(C^{\diamond}\right)\right\rangle\right\rangle$.

We first compute

$$
\begin{aligned}
& \tau=\left(\begin{array}{cc}
\sin ^{2} \delta+(1-p) \cos ^{2} \delta & 0 \\
0 & p \cos ^{2} \delta
\end{array}\right) \approx\left(\begin{array}{cc}
1-p & 0 \\
0 & p
\end{array}\right) \\
& \tau^{\prime}=\left(\begin{array}{cc}
\sin ^{2} \delta-(1-p) \cos ^{2} \delta & 0 \\
0 & -p \cos ^{2} \delta
\end{array}\right) \approx\left(\begin{array}{cc}
-(1-p) & 0 \\
0 & -p
\end{array}\right) \\
& \tilde{\tau}=\left(\begin{array}{cc}
\operatorname{Tr}\left[\tilde{C}\left(\begin{array}{cc}
1 & 0 \\
0 & 1-p
\end{array}\right)\right] & \operatorname{Tr}\left[\tilde{C}\left(\begin{array}{cc}
0 & \sqrt{p} \\
0 & 0
\end{array}\right)\right] \\
\operatorname{Tr}\left[\tilde{C}\left(\begin{array}{cc}
0 & 0 \\
\sqrt{p} & 0
\end{array}\right)\right] & \operatorname{Tr}\left[\tilde{C}\left(\begin{array}{cc}
0 & 0 \\
0 & p
\end{array}\right)\right]
\end{array}\right),
\end{aligned}
$$

where by " $\approx$ " we ignore the small contribution of $O(\delta)$. Then we have

$$
f_{2}\left(C^{\diamond}\right) \approx-|00\rangle
$$

$$
\begin{aligned}
& f_{3}\left(C^{\diamond}\right)^{-1} \approx p|01\rangle\langle 01|+p|10\rangle\langle 10| \\
&+(|00\rangle+(1-p)|11\rangle)(\langle 00|+(1-p)\langle 11|) \frac{1}{2(1-p)}+(p|11\rangle)(p\langle 11|) \frac{1}{2 p} \\
& f_{3}\left(C^{\diamond}\right)^{-1} \approx \frac{1}{p}|01\rangle\left\langle 01\left|+\frac{1}{p}\right| 10\right\rangle\langle 10|+\frac{2}{p}[(1-p)|00\rangle\langle 00| \\
&+|11\rangle\langle 11|-(1-p)| 00\rangle\langle 11|-(1-p)| 11\rangle\langle 00|] .
\end{aligned}
$$

Then $\left.\left|\tilde{C}^{\diamond}\right\rangle\right\rangle \approx|00\rangle-|11\rangle$ and we can take $D^{\diamond}=\cos \delta|0\rangle\langle 0|-\sin \delta| 1\rangle\langle 1|$. 


\section{Attaining the asymptotic QFI}

Now we have the optimal code from the previous two steps, i.e.,

$$
\begin{aligned}
\left|0_{L}\right\rangle & =\sin (\delta+\varepsilon)|0\rangle_{\mathcal{P}}|00\rangle_{\mathcal{A}}+\cos (\delta+\varepsilon)|1\rangle_{\mathcal{P}}|10\rangle_{\mathcal{A}}, \\
\left|1_{L}\right\rangle & =\sin (\delta-\varepsilon)|0\rangle_{\mathcal{P}}|01\rangle_{\mathcal{A}}+\cos (\delta-\varepsilon)|1\rangle_{\mathcal{P}}|11\rangle_{\mathcal{A}},
\end{aligned}
$$

where $\delta$ and $\varepsilon=o(\delta)$ are small values. The last step is to find the exact relation between $\delta$ and $\varepsilon$ and $\mathfrak{F}_{\mathrm{SQL}}\left(\mathcal{N}_{\omega}^{\mathrm{AD}}\right)-$ $\mathfrak{F}_{\mathrm{SOL}}\left(\mathcal{D}_{L, \omega}\right)$.

To this end, we need the near-optimal recovery channel computed using Eq. (40)

$$
G_{\mathrm{opt}}=\frac{2 i}{\sqrt{1-p}}|00\rangle\left\langle 11\left|+\frac{-2 i}{\sqrt{1-p}}\right| 11\right\rangle\langle 00|:=\left(\begin{array}{cccc}
0 & 0 & 0 & \frac{2 i}{\sqrt{1-p}} \\
0 & 0 & 0 & 0 \\
0 & 0 & 0 & 0 \\
\frac{-2 i}{\sqrt{1-p}} & 0 & 0 & 0
\end{array}\right)
$$

and

$$
T_{\mathrm{opt}}=e^{i \varepsilon G_{\mathrm{opt}}}=\left(\begin{array}{cccc}
\cos \left(\frac{2 \varepsilon}{\sqrt{1-p}}\right) & 0 & 0 & -\sin \left(\frac{2 \varepsilon}{\sqrt{1-p}}\right) \\
0 & 1 & 0 & 0 \\
0 & 0 & 1 & 0 \\
\sin \left(\frac{2 \varepsilon}{\sqrt{1-p}}\right) & 0 & 0 & \cos \left(\frac{2 \varepsilon}{\sqrt{1-p}}\right)
\end{array}\right)
$$

Then, using Eq. (38) and

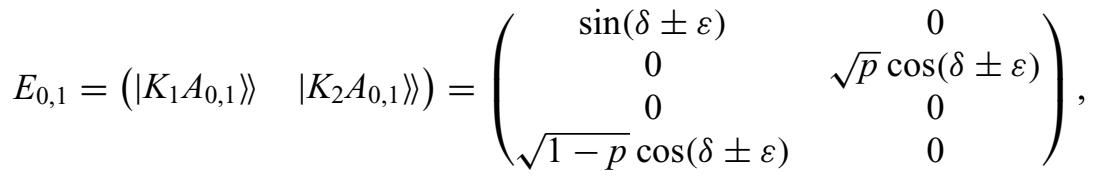

we finally obtain

$$
\begin{aligned}
\xi= & p[\cos (2 \delta)+\cos (2 \varepsilon)] \sin ^{2}\left(\frac{\varepsilon}{\sqrt{1-p}}\right) \\
& +\sqrt{1-p} \sin (2 \varepsilon) \sin \left(\frac{2 \varepsilon}{\sqrt{1-p}}\right)+\cos (2 \varepsilon) \cos \left(\frac{2 \varepsilon}{\sqrt{1-p}}\right) \\
= & 1-\frac{2 p \sin ^{2} \delta}{1-p} \varepsilon^{2}+O\left(\varepsilon^{4}\right), \\
\dot{\xi}= & -i \sqrt{1-p} \sin (2 \delta) \sin \left(\frac{2 \varepsilon}{\sqrt{1-p}}\right)=-2 i \sin (2 \delta) \varepsilon+O\left(\varepsilon^{3}\right) .
\end{aligned}
$$

Clearly,

$$
\mathfrak{F}_{\mathrm{SQL}}\left(\mathcal{D}_{L, \omega}\right)=\frac{4(1-p) \cos ^{2} \delta}{p}+O\left(\varepsilon^{2}\right)
$$

as expected. When $\delta$ is small and $\varepsilon=o(\delta)$, we have $\mathfrak{F}_{\mathrm{SQL}}\left(\mathcal{D}_{L, \omega}\right) \approx \mathfrak{F}_{\mathrm{SQL}}\left(\mathcal{N}_{\omega}^{\mathrm{AD}}\right)$. 
[1] Vittorio Giovannetti, Seth Lloyd, and Lorenzo Maccone, Advances in quantum metrology, Nat. Photonics 5, 222 (2011).

[2] C. L. Degen, F. Reinhard, and P. Cappellaro, Quantum sensing, Rev. Mod. Phys. 89, 035002 (2017).

[3] Daniel Braun, Gerardo Adesso, Fabio Benatti, Roberto Floreanini, Ugo Marzolino, Morgan W. Mitchell, and Stefano Pirandola, Quantum-enhanced measurements without entanglement, Rev. Mod. Phys. 90, 035006 (2018).

[4] Luca Pezzè, Augusto Smerzi, Markus K. Oberthaler, Roman Schmied, and Philipp Treutlein, Quantum metrology with nonclassical states of atomic ensembles, Rev. Mod. Phys. 90, 035005 (2018).

[5] Stefano Pirandola, Bhaskar Roy Bardhan, Tobias Gehring, Christian Weedbrook, and Seth Lloyd, Advances in photonic quantum sensing, Nat. Photonics. 12, 724 (2018).

[6] B. C. Sanders and G. J. Milburn, Optimal Quantum Measurements for Phase Estimation, Phys. Rev. Lett. 75, 2944 (1995).

[7] J. J. Bollinger, Wayne M. Itano, D. J. Wineland, and D. J. Heinzen, Optimal frequency measurements with maximally correlated states, Phys. Rev. A 54, R4649 (1996).

[8] S. F. Huelga, C. Macchiavello, T. Pellizzari, A. K. Ekert, M. B. Plenio, and J. I. Cirac, Improvement of Frequency Standards with Quantum Entanglement, Phys. Rev. Lett. 79, 3865 (1997).

[9] D. Leibfried, M. D. Barrett, T. Schaetz, J. Britton, J. Chiaverini, W. M. Itano, J. D. Jost, C. Langer, and D. J. Wineland, Toward heisenberg-limited spectroscopy with multiparticle entangled states, Science 304, 1476 (2004).

[10] Carlton M. Caves, Quantum-mechanical noise in an interferometer, Phys. Rev. D 23, 1693 (1981).

[11] Bernard Yurke, Samuel L. McCall, and John R. Klauder, $\mathrm{Su}(2)$ and $\mathrm{su}(1,1)$ interferometers, Phys. Rev. A 33, 4033 (1986).

[12] D. W. Berry and H. M. Wiseman, Optimal States and Almost Optimal Adaptive Measurements for Quantum Interferometry, Phys. Rev. Lett. 85, 5098 (2000).

[13] Brendon L. Higgins, Dominic W. Berry, Stephen D. Bartlett, Howard M. Wiseman, and Geoff J. Pryde, Entanglement-free heisenberg-limited phase estimation, Nature 450, 393 (2007).

[14] V. Buzek, R. Derka, and S. Massar, Optimal Quantum Clocks, Phys. Rev. Lett. 82, 2207 (1999).

[15] Wojciech Górecki, Rafa Demkowicz-Dobrzanśki, Howard M. Wiseman, and Dominic W. Berry, $\pi$-corrected Heisenberg Limit, Phys. Rev. Lett. 124, 030501 (2020).

[16] Vittorio Giovannetti, Seth Lloyd, and Lorenzo Maccone, Quantum-enhanced measurements: Beating the standard quantum limit, Science 306, 1330 (2004).

[17] Alejandra Valencia, Giuliano Scarcelli, and Yanhua Shih, Distant clock synchronization using entangled photon pairs, Appl. Phys. Lett. 85, 2655 (2004).

[18] Mark de Burgh and Stephen D. Bartlett, Quantum methods for clock synchronization: Beating the standard quantum limit without entanglement, Phys. Rev. A 72, 042301 (2005).

[19] Carl Wilhelm Helstrom, Quantum Detection and Estimation Theory (Academic Press, New York, 1976).
[20] Alexander S. Holevo, Probabilistic and Statistical Aspects of Quantum Theory (Springer Science \& Business Media, Berlin, 2011), Vol. 1

[21] Matteo G. A. Paris, Quantum estimation for quantum technology, Int. J. Quantum Inf. 7, 125 (2009).

[22] Samuel L. Braunstein and Carlton M. Caves, Statistical Distance and the Geometry of Quantum States, Phys. Rev. Lett. 72, 3439 (1994).

[23] Vittorio Giovannetti, Seth Lloyd, and Lorenzo Maccone, Quantum Metrology, Phys. Rev. Lett. 96, 010401 (2006).

[24] Akio Fujiwara and Hiroshi Imai, A fibre bundle over manifolds of quantum channels and its application to quantum statistics, J. Phys. A: Math. Theor. 41, 255304 (2008).

[25] Z. Ji, G. Wang, R. Duan, Y. Feng, and M. Ying, Parameter estimation of quantum channels, IEEE Trans. Inf. Theory 54, 5172 (2008).

[26] B. M. Escher, R. L. de Matos Filho, and L. Davidovich, General framework for estimating the ultimate precision limit in noisy quantum-enhanced metrology, Nat. Phys. 7, 406 (2011).

[27] Masahito Hayashi, Comparison between the cramer-rao and the mini-max approaches in quantum channel estimation, Commun. Math. Phys. 304, 689 (2011).

[28] Rafa Demkowicz-Dobrzański, Jan Koodyński, and Madalin Guta, The elusive heisenberg limit in quantumenhanced metrology, Nat. Commun. 3, 1063 (2012).

[29] Jan Koodyński and Rafa Demkowicz-Dobrzański, Efficient tools for quantum metrology with uncorrelated noise, New J. Phys. 15, 073043 (2013).

[30] Sergey I. Knysh, Edward H. Chen, and Gabriel A. Durkin, True limits to precision via unique quantum probe, arXiv:1402.0495 [quant-ph] (2014).

[31] Rafa Demkowicz-Dobrzanśki and Lorenzo Maccone, Using Entanglement against Noise in Quantum Metrology, Phys. Rev. Lett. 113, 250801 (2014).

[32] Pavel Sekatski, Michalis Skotiniotis, Janek Koodyński, and Wolfgang Dür, Quantum metrology with full and fast quantum control, Quantum 1, 27 (2017).

[33] Rafa Demkowicz-Dobrzanśki, Jan Czajkowski, and Pavel Sekatski, Adaptive Quantum Metrology under General Markovian Noise, Phys. Rev. X 7, 041009 (2017).

[34] Sisi Zhou, Mengzhen Zhang, John Preskill, and Liang Jiang, Achieving the heisenberg limit in quantum metrology using quantum error correction, Nat. Commun. 9, 78 (2018).

[35] Haidong Yuan and Chi-Hang Fred Fung, Fidelity and fisher information on quantum channels, New J. Phys. 19, 113039 (2017).

[36] Vishal Katariya and Mark M. Wilde, Geometric distinguishability measures limit quantum channel estimation and discrimination, arXiv:2004.10708 [quant-ph] (2020).

[37] Haidong Yuan and Chi-Hang Fred Fung, Quantum parameter estimation with general dynamics, npj Quantum Inf. 3, 1 (2017).

[38] D. J. Wineland, J. J. Bollinger, W. M. Itano, F. L. Moore, and D. J. Heinzen, Spin squeezing and reduced quantum noise in spectroscopy, Phys. Rev. A 46, R6797 (1992).

[39] Duger Ulam-Orgikh and Masahiro Kitagawa, Spin squeezing and decoherence limit in ramsey spectroscopy, Phys. Rev. A 64, 052106 (2001). 
[40] Rafa Demkowicz-Dobrzanśki, Konrad Banaszek, and Roman Schnabel, Fundamental quantum interferometry bound for the squeezed-light-enhanced gravitational wave detector geo 600, Phys. Rev. A 88, 041802 (2013).

[41] R. Chaves, J. B. Brask, M. Markiewicz, J. Koodynśki, and A. Acń, Noisy Metrology beyond the Standard Quantum Limit, Phys. Rev. Lett. 111, 120401 (2013).

[42] Tuvia Gefen, David A. Herrera-Martí, and Alex Retzker, Parameter estimation with efficient photodetectors, Phys. Rev. A 93, 032133 (2016).

[43] Martin B. Plenio and Susana F. Huelga, Sensing in the presence of an observed environment, Phys. Rev. A 93, 032123 (2016).

[44] Francesco Albarelli, Matteo A. C. Rossi, Matteo G. A. Paris, and Marco G. Genoni, Ultimate limits for quantum magnetometry via time-continuous measurements, New J. Phys. 19, 123011 (2017).

[45] Francesco Albarelli, Matteo A. C. Rossi, Dario Tamascelli, and Marco G. Genoni, Restoring heisenberg scaling in noisy quantum metrology by monitoring the environment, Quantum 2, 110 (2018).

[46] Yuichiro Matsuzaki, Simon C. Benjamin, and Joseph Fitzsimons, Magnetic field sensing beyond the standard quantum limit under the effect of decoherence, Phys. Rev. A 84, 012103 (2011).

[47] Alex W. Chin, Susana F. Huelga, and Martin B. Plenio, Quantum Metrology in Non-Markovian Environments, Phys. Rev. Lett. 109, 233601 (2012).

[48] Andrea Smirne, Jan Koodynśki, Susana F. Huelga, and Rafa Demkowicz-Dobrzanśki, Ultimate Precision Limits for Noisy Frequency Estimation, Phys. Rev. Lett. 116, 120801 (2016).

[49] Jing Liu and Haidong Yuan, Quantum parameter estimation with optimal control, Phys. Rev. A 96, 012117 (2017).

[50] Han Xu, Junning Li, Liqiang Liu, Yu Wang, Haidong Yuan, and Xin Wang, Generalizable control for quantum parameter estimation through reinforcement learning, npj Quantum Inf. 5, 1 (2019).

[51] Krzysztof Chabuda, Jacek Dziarmaga, Tobias J. Osborne, and Rafa Demkowicz-Dobrzański, Tensor-network approach for quantum metrology in many-body quantum systems, Nat. Commun. 11, 1 (2020).

[52] Sisi Zhou and Liang Jiang, Optimal approximate quantum error correction for quantum metrology, Phys. Rev. Res. 2, 013235 (2020).

[53] Masahiro Hotta, Tokishiro Karasawa, and Masanao Ozawa, Ancilla-assisted enhancement of channel estimation for low-noise parameters, Phys. Rev. A 72, 052334 (2005).

[54] M. Hotta, T. Karasawa, and M. Ozawa, N-body-extended channel estimation for low-noise parameters, J. Phys. A: Math. Gen. 39, 14465 (2006).

[55] Stefano Pirandola, Riccardo Laurenza, Carlo Ottaviani, and Leonardo Banchi, Fundamental limits of repeaterless quantum communications, Nat. Commun. 8, 1 (2017).

[56] Stefano Pirandola and Cosmo Lupo, Ultimate Precision of Adaptive Noise Estimation, Phys. Rev. Lett. 118, 100502 (2017).
[57] Masahiro Takeoka and Mark M. Wilde, Optimal estimation and discrimination of excess noise in thermal and amplifier channels, arXiv:1611.09165 [quant-ph] (2016).

[58] Riccardo Laurenza, Cosmo Lupo, Gaetana Spedalieri, Samuel L. Braunstein, and Stefano Pirandola, Channel simulation in quantum metrology, Quantum Meas. Quantum Metrol. 5, 1 (2018).

[59] Daniel A. Lidar and Todd A. Brun, Quantum Error Correction (Cambridge University Press, Cambridge, 2013).

[60] Emanuel Knill and Raymond Laflamme, Theory of quantum error-correcting codes, Phys. Rev. A 55, 900 (1997).

[61] Daniel Gottesman, in Quantum Information Science and its Contributions to Mathematics, Proceedings of Symposia in Applied Mathematics Vol. 68 (2010), p. 13.

[62] Wolfgang Dür and Hans J. Briegel, Entanglement purification and quantum error correction, Rep. Prog. Phys. 70, 1381 (2007).

[63] E. M. Kessler, I. Lovchinsky, A. O. Sushkov, and M. D. Lukin, Quantum Error Correction for Metrology, Phys. Rev. Lett. 112, 150802 (2014).

[64] G. Arrad, Y. Vinkler, D. Aharonov, and A. Retzker, Increasing Sensing Resolution with Error Correction, Phys. Rev. Lett. 112, 150801 (2014).

[65] W. Dür, M. Skotiniotis, F. Fröwis, and B. Kraus, Improved Quantum Metrology Using Quantum Error Correction, Phys. Rev. Lett. 112, 080801 (2014).

[66] Roee Ozeri, Heisenberg limited metrology using quantum error-correction codes, arXiv:1310.3432 [quant-ph] (2013).

[67] Thomas Unden, Priya Balasubramanian, Daniel Louzon, Yuval Vinkler, Martin B. Plenio, Matthew Markham, Daniel Twitchen, Alastair Stacey, Igor Lovchinsky, Alexander O. Sushkov, Mikhail D. Lukin, Alex Retzker, Boris Naydenov, Liam P. McGuinness, and Fedor Jelezko, Quantum Metrology Enhanced by Repetitive Quantum Error Correction, Phys. Rev. Lett. 116, 230502 (2016).

[68] Florentin Reiter, Anders Sndberg Srensen, Peter Zoller, and C. A. Muschik, Dissipative quantum error correction and application to quantum sensing with trapped ions, Nat. Commun. 8, 1822 (2017).

[69] Xiao-Ming Lu, Sixia Yu, and C. H. Oh, Robust quantum metrological schemes based on protection of quantum fisher information, Nat. Commun. 6, 7282 (2015).

[70] David Layden and Paola Cappellaro, Spatial noise filtering through error correction for quantum sensing, npj Quantum Inf. 4, 30 (2018).

[71] David Layden, Sisi Zhou, Paola Cappellaro, and Liang Jiang, Ancilla-Free Quantum Error Correction Codes for Quantum Metrology, Phys. Rev. Lett. 122, 040502 (2019).

[72] Wojciech Gorecki, Sisi Zhou, Liang Jiang, and Rafa Demkowicz-Dobrzański, Optimal probes and errorcorrection schemes in multi-parameter quantum metrology, arXiv:1901.00896 [quant-ph] (2019).

[73] Kok Chuan Tan, S. Omkar, and Hyunseok Jeong, Quantum-error-correction-assisted quantum metrology without entanglement, Phys. Rev. A 100, 022312 (2019).

[74] Theodoros Kapourniotis and Animesh Datta, Faulttolerant quantum metrology, Phys. Rev. A 100, 022335 (2019). 
[75] Quntao Zhuang, John Preskill, and Liang Jiang, Distributed quantum sensing enhanced by continuousvariable error correction, New J. Phys. 22, 022001 (2020).

[76] Yu Chen, Hongzhen Chen, Jing Liu, Zibo Miao, and Haidong Yuan, Fluctuation-enhanced quantum metrology, arXiv:2003.13010 [quant-ph] (2020).

[77] George Casella and Roger L. Berger, Statistical Inference (Duxbury Press, Duxbury Pacific Grove, CA, 2002), Vol. 2.

[78] Erich L. Lehmann and George Casella, Theory of Point Estimation (Springer Science \& Business Media, Berlin, 2006).

[79] O. E. Barndorff-Nielsen and R. D. Gill, Fisher information in quantum statistics, J. Phys. A: Math. Gen. 33, 4481 (2000).

[80] Richard D. Gill and Serge Massar, State estimation for large ensembles, Phys. Rev. A 61, 042312 (2000).

[81] Sean D. Huver, Christoph F. Wildfeuer, and Jonathan P. Dowling, Entangled fock states for robust quantum optical metrology, imaging, and sensing, Phys. Rev. A 78, 063828 (2008).

[82] R. Demkowicz-Dobrzanśki, U. Dorner, B. J. Smith, J. S. Lundeen, W. Wasilewski, K. Banaszek, and I. A. Walmsley, Quantum phase estimation with lossy interferometers, Phys. Rev. A 80, 013825 (2009).

[83] Masahito Hayashi, Two quantum analogues of fisher information from a large deviation viewpoint of quantum estimation, J. Phys. A: Math. Gen. 35, 7689 (2002).

[84] Stefano Pirandola, Riccardo Laurenza, Cosmo Lupo, and Jason L. Pereira, Fundamental limits to quantum channel discrimination, npj Quantum Inf. 5, 1 (2019).

[85] Yu Chen and Haidong Yuan, Zero-error quantum hypothesis testing in finite time with quantum error correction, Phys. Rev. A 100, 022336 (2019).

[86] Giulio Chiribella, Giacomo M. D'Ariano, and Paolo Perinotti, Memory Effects in Quantum Channel Discrimination, Phys. Rev. Lett. 101, 180501 (2008).

[87] Yuxiang Yang, Memory Effects in Quantum Metrology, Phys. Rev. Lett. 123, 110501 (2019).

[88] M. Hayashi, Discrimination of two channels by adaptive methods and its application to quantum system, IEEE Trans. Inf. Theory 55, 3807 (2009).

[89] Tom Cooney, Milán Mosonyi, and Mark M. Wilde, Strong converse exponents for a quantum channel discrimination problem and quantum-feedback-assisted communication, Commun. Math. Phys. 344, 797 (2016).

[90] Mark M. Wilde, Mario Berta, Christoph Hirche, and Eneet Kaur, Amortized channel divergence for asymptotic quantum channel discrimination, Lett. Math. Phys. 110, 2277 (2020).

[91] Xin Wang and Mark M. Wilde, Resource theory of asymmetric distinguishability for quantum channels, Phys. Rev. Res. 1, 033169 (2019).
[92] Kun Fang, Omar Fawzi, Renato Renner, and David Sutter, Chain Rule for the Quantum Relative Entropy, Phys. Rev. Lett. 124, 100501 (2020).

[93] Luca Pezzé and Augusto Smerzi, Entanglement, Nonlinear Dynamics, and the Heisenberg Limit, Phys. Rev. Lett. 102, 100401 (2009).

[94] Masahiro Kitagawa and Masahito Ueda, Squeezed spin states, Phys. Rev. A 47, 5138 (1993).

[95] Akio Fujiwara and Hiroshi Imai, Quantum parameter estimation of a generalized pauli channel, J. Phys. A, Math. Gen. 36, 8093 (2003).

[96] Hidetoshi Komiya, Elementary proof for sion's minimax theorem, Kodai Math. J. 11, 5 (1988).

[97] Maria do Rosário Grossinho and Stepan Agop Tersian, An Introduction to Minimax Theorems and Their Applications to Differential Equations (Springer Science \& Business Media, Berlin, 2001), Vol. 52.

[98] Stephen Boyd and Lieven Vandenberghe, Convex Optimization (Cambridge University Press, Cambridge, 2004).

[99] John Watrous, The Theory of Quantum Information (Cambridge University Press, Cambridge, 2018).

[100] U. Dorner, Quantum frequency estimation with trapped ions and atoms, New J. Phys. 14, 043011 (2012).

[101] Sisi Zhou, Zi-Wen Liu, and Liang Jiang, New perspectives on covariant quantum error correction, arXiv:2005.119 18v2 [quant-ph] (2020).

[102] Christoph Dankert, Efficient simulation of random quantum states and operators, arXiv:quant-ph/0512217 [quantph] (2005).

[103] Giulio Chiribella, Giacomo Mauro D'Ariano, and Paolo Perinotti, Realization schemes for quantum instruments in finite dimensions, J. Math. Phys. 50, 042101 (2009).

[104] M. M. Wilde, M. Tomamichel, and M. Berta, Converse bounds for private communication over quantum channels, IEEE Trans. Inf. Theory 63, 1792 (2017).

[105] Aleksander Kubica and Rafa Demkowicz-Dobrzański, Using quantum metrological bounds in quantum error correction: A simple proof of the approximate eastin-knill theorem, arXiv:2004.11893 [quant-ph] (2020).

[106] Raphael Kaubruegger, Pietro Silvi, Christian Kokail, Rick van Bijnen, Ana Maria Rey, Jun Ye, Adam M. Kaufman, and Peter Zoller, Variational Spin-Squeezing Algorithms on Programmable Quantum Sensors, Phys. Rev. Lett. 123, 260505 (2019).

[107] Bálint Koczor, Suguru Endo, Tyson Jones, Yuichiro Matsuzaki, and Simon C. Benjamin, Variational-state quantum metrology, New J. Phys. 22, 083038 (2020).

[108] Johannes Jakob Meyer, Johannes Borregaard, and Jens Eisert, A variational toolbox for quantum multi-parameter estimation, arXiv:2006.06303 [quant-ph] (2020).

[109] Haidong Yuan, Sequential Feedback Scheme Outperforms the Parallel Scheme for Hamiltonian Parameter Estimation, Phys. Rev. Lett. 117, 160801 (2016). 